Discussion Paper No. 14-056

\title{
Voting for Burden Sharing Rules in Public Goods Games
}

\author{
Carlo Gallier, Martin Kesternich, \\ and Bodo Sturm
}

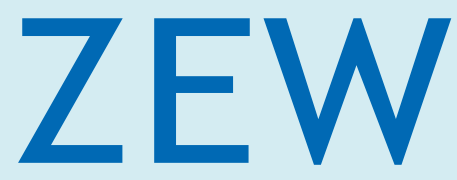

Zentrum für Europäische Wirtschaftsforschung $\mathrm{GmbH}$

Centre for European Economic Research 
Discussion Paper No. 14-056

\title{
Voting for Burden Sharing Rules in Public Goods Games
}

\author{
Carlo Gallier, Martin Kesternich, \\ and Bodo Sturm
}

Download this ZEW Discussion Paper from our ftp server:

http://ftp.zew.de/pub/zew-docs/dp/dp14056.pdf

Die Discussion Papers dienen einer möglichst schnellen Verbreitung von neueren Forschungsarbeiten des ZEW. Die Beiträge liegen in alleiniger Verantwortung der Autoren und stellen nicht notwendigerweise die Meinung des ZEW dar.

Discussion Papers are intended to make results of ZEW research promptly available to other economists in order to encourage discussion and suggestions for revisions. The authors are solely responsible for the contents which do not necessarily represent the opinion of the ZEW. 


\title{
Voting for Burden Sharing Rules in Public Goods Games
}

\author{
Carlo Gallier ${ }^{*}$, Martin Kesternich* and Bodo Sturm ${ }^{*}{ }^{*}$ \\ ${ }^{*}$ Centre for European Economic Research \\ ${ }^{\dagger}$ Leipzig University of Applied Sciences
}

Version: August 27, 2014

\begin{abstract}
:
In this experiment, we endogenize the choice of which contribution scheme is implemented in a public goods game. We investigate three rule-based contribution schemes. In a first step, players agree on a common group provision level using the principle of the smallest common denominator. Subsequently, this group investment is allocated according to a specific rule to individual minimum contributions. The game is implemented either as a Single- or a MultiPhase Game. In the Single-Phase Game, the contribution schemes are exogenously implemented. In the Multi-Phase Game, we let subjects vote on the rule-based contribution schemes. If a scheme obtains a sufficient majority it is implemented. In case no sufficient majority is reached, subjects have to make their contributions to the public good using the voluntary contribution mechanism (VCM). Our results suggest that the endogenous choice of a contribution scheme has an impact on the level of contributions. In case of a rule-based contribution scheme which equalizes payoffs, contributions are higher if subjects choose the scheme than in case the scheme is implemented exogenously. In contrast, contributions are higher if the VCM is implemented exogenously than in case a sufficient majority cannot be obtained and, therefore, subjects have to play the VCM.
\end{abstract}

Keywords: public goods, endogenous institutions, minimum contribution rules, cooperation

JEL: C72, C92, H41

Acknowledgement: Financial support by the German Federal Ministry of Education and Research (FKZ 01UN1016A) is gratefully acknowledged. We thank the MaXLab team at the University of Magdeburg for their support.

Correspondence: Carlo Gallier (gallier@zew.de), Martin Kesternich (kesternich@zew.de), Bodo Sturm (bodo.sturm@htwk-leipzig.de) 


\section{Introduction}

Inducing contributions to public goods remains an important endeavor and is particularly demanding when sovereign agents have different interests due to heterogeneous preferences. Free-riding incentives prevent the voluntary provision of the public good and any institutional design which aims at countervailing free riding has to address the question of a fair distribution of the costs and benefits of providing public goods. The experimental literature suggests rule-based contribution schemes that are inspired by different fairness norms to be effective in enhancing cooperation (e.g., Dannenberg et al. 2014, Kesternich et al. 2014a,b, Orzen 2008). In such a scheme, agents have to agree upon a common group provision level of the public good in combination with a burden sharing rule, i.e., a rule how to share the associated costs among the involved parties. All previous papers devoted to this issue assume burden sharing rules to be exogenously imposed. We argue that an external implementation is not always a feasible or desirable option due to sovereign interests. Furthermore, owing to heterogeneous preferences a variety of burden sharing rules appears to be plausible. It is therefore an essential challenge for groups of sovereign and heterogeneous agents to endogenously determine their institutional framework for the provision of public goods in terms of a burden sharing agreement.

In this paper, we endogenize the decision on how to share the burden in a public goods game and explore the performance of different rule-based contribution schemes when sovereign agents differ with respect to their initial wealth position. More precisely, we contribute to the following research questions: (i) Which burden sharing rule do agents actually prefer; (ii) Are sovereign and heterogeneous agents able to agree upon a common burden sharing rule; and (iii) Does the endogenous choice of a contribution scheme affect the level of private contributions to the public good?

In our experimental setting, all rule-based contribution schemes are based on the principle of the "smallest common denominator" and include two steps: Firstly, all agents can propose a minimum group contribution level to the public good. Then, the minimum of all proposals is selected and allocated across subjects according to a pre-defined burden sharing rule. This approach reflects many real world institutional arrangements for local and global public goods that both involve the choice of a provision goal and a burden sharing rule. Referring to climate policy, for example, a pre-negotiated rule such as equal carbon reductions among countries (Barrett 2003) may be particularly beneficial in reducing negotiation costs when the total reduction target changes over time. Since each participating country needs to sign and ratify the agreement, the player with the smallest proposal is pivotal. Countries can, however, 
voluntarily go beyond their obligations. ${ }^{1}$ In the context of small-scale common-pool resources, in contrast, Chambers (1980) and Ostrom (1990) postulate that governing schemes which are characterized by equivalent rules for distributing the costs and benefits lead to comparatively high cooperation levels. Dayton-Johnson (2000a,b) provides a more rigorous theoretical and empirical analysis and concludes that institutions with congruence between the distribution of costs and benefits are comparatively successful in managing local commons. However, there are different rules how to share the costs and benefits of providing a public good and it is a crucial step for the agents to agree upon a common rule how to distribute the associated costs and benefits. Thinking of international climate negotiations, for example, a variety of pre-negotiated rules for carbon emissions reductions among participating countries is conceivable and it is a major challenge for participants to agree upon a common rule (Lange et al. 2007,2010, Kesternich et al. 2014c). Preferences for burden sharing rules may be inspired by different notions on distributive justice such as accountability, efficiency, need, and equality (Johansson-Stenman and Konow 2010). For instance, the need principle requires the satisfaction of basic needs such as food, shelter, and clothes and therefore shifts the burden towards agents with substantial economic capacities. This approach corresponds to Mill's concept of “equality of sacrifice” (Mill 1848) being initially discussed as a principle for tax distribution with the aim of harmonizing payoffs among individuals (ability-to-pay rule). In contrast, calls for equal percentage reductions of emissions (sovereignty or grandfathering rule) may be rather consistent with a fairness norm which requires equal contributions from all agents.

Our three-player repeated public goods game is implemented either as a Single- or a MultiPhase Game. Subjects in the Multi-Phase Game play a collective-choice and a contribution phase. In the collective-choice phase, subjects vote upon a common burden sharing rule either by majority or unanimity rule voting. The burden sharing rules are inspired by different fairness norms and include equal contributions from all group members to the public good (eqcont), equal payoffs among all group members (eqpay) and contributions to the public good that are proportional to participants’ initial endowment (propcont). If a burden sharing rule obtains a sufficient majority it is implemented in the contribution phase. In case no sufficient majority is reached, subjects have to make their contributions to the public good using the voluntary contribution mechanism (VCM). In the Single-Phase Game, subjects play

\footnotetext{
${ }^{1}$ First applications of the principle of the smallest common denominator in the context of environmental agreements can be found in Endres (1997) and Endres and Finus (1999).
} 
only the contribution phase and make their contributions to the public good according to the exogenously implemented contribution schemes.

Our experimental results suggest negotiations upon a common burden sharing agreement to significantly affect individual contributions to public goods. Most importantly, in case of a scheme aiming at equalizing payoffs, contributions are higher if this allocation rule is endogenously implemented. The contrary holds for the VCM. If exogenously implemented, contributions are higher in the VCM than in the case where subjects fail to achieve an agreement and therefore end up in an uncoordinated action (VCM). Our results confirm previous experimental evidence showing different rule-based contribution schemes to be effective in increasing contribution levels in public goods games when subjects differ with respect to their initial endowment. Endogenously or exogenously implemented, these allocation rules counteract typical downward trends in public good provision levels in absence of any institutional framework. There is, however, evidence that preferences for different allocation rules depend on the individual wealth position within the group. The higher subject's initial endowment, the less (more) frequently they vote for the eqpay (eqcont) scheme. Nevertheless, we find subjects to largely recognize the potential efficiency gains of the different allocation rules and ending up in a successful burden sharing agreement.

The remainder of the paper is organized as follows: Section 2 provides an overview of the related literature. Section 3 describes the experimental design and derives our theoretical predictions. We present the experimental results in Section 4 and Section 5 concludes the paper.

\section{A Brief Review of Related Literature}

Two strands of the experimental literature are important for our research questions. Firstly, there is vibrant literature on the effect of rule-based contribution schemes on the voluntary provision of public goods. In such a contribution scheme, in a first step players agree on a common group provision level with the underlying principle of the smallest common denominator. In a second step, this group investment is allocated according to a specific burden sharing rule to derive individual minimum contributions. Rule-based contribution schemes provide an effective way to tackle the public goods' free-rider problem by transforming the social dilemma situation of the VCM into a game with equilibria with positive contributions. Orzen (2008) shows rule-based contribution schemes to be effective in enhancing cooperation gains in homogeneous public good settings. Dannenberg et al. (2014) experimentally study the voluntary formation of coalitions to provide public goods. 
Participation and commitment in the coalition are either exogenously imposed or endogenously determined by the players themselves. They report participation rates to be larger when commitments in the coalition are endogenously determined using a minimum contribution rule rather than exogenously determined. While in case of homogeneous groups, the only fair burden sharing rule assigns the same burden to all agents. In a heterogeneous setting different contribution schemes - inspired by different fairness norms - may seem plausible. Kesternich et al. (2014a) find that rule-based contribution schemes lead to substantial cooperation gains if agents differ in their benefits from the public good. They observe that a burden sharing rule that aims at equalizing payoffs by explicitly addressing redistribution among heterogeneous agents payoff-dominates all other burden sharing mechanisms. Coordination within the different burden sharing schemes is particularly challenging if agents differ with respect to their initial endowments and further experimental investigation suggests the perception on various contribution norms to be sensitive to the circumstances under which the different positions accrue (Kesternich et al. 2014b).

In all these experiments, the burden sharing rules are exogenously imposed but an external implementation cannot always be considered as a feasible option among sovereign agents nor may it be desirable in all cases. This brings us to a further relevant strand of literature: The growing number of experiments on the effects of endogenous institutions in social dilemma situations. Experimental findings suggest that the endogenous choice of an institutional setting has a positive impact on cooperation levels. ${ }^{2}$ Walker et al. (2000) and Margreiter et al. (2005) use a common-pool resource experiment and investigate the effect of jointly determined appropriation levels in contrast to independent and individual appropriation decisions. In a homogeneous setting, Walker et al. (2000) examine a two-stage game consisting of a collective-choice and an individual-contribution stage. In the collective-choice stage, each subject can propose appropriation levels for the common-pool resource for each group member, followed by the opportunity to vote anonymously on the proposals. If any proposal obtains a sufficient majority, the proposal is implemented in the contribution stage of the game. In case a majority cannot be obtained, group members make their appropriation decision independently. They report participants to be more cooperative in case of a

\footnotetext{
${ }^{2}$ For further experimental evidence see Kosfeld et al. (2009), Ertan et al. (2009), and Hamman et al. (2011). Kosfeld et al. (2009) investigate the endogenous formation of institutions in public goods provision and conclude that institutions are formed and that they positively affect cooperation. Ertan et al. (2009) study a repeated public goods game in which punishment may be allowed, depending on subjects' votes. They find an evolution towards allowing punishment of low contributions. In Hamman et al. (2011), each group selects an "allocator" at the beginning of each round of a repeated public goods game. The allocator then chooses a vector of contributions. The result is that the delegation increases the contributions.
} 
successfully adopted agreement in contrast to an uncoordinated action. Margreiter et al. (2005) extend this mechanism to a setting with heterogeneous agents. Their results show that heterogeneity makes it more difficult to achieve an agreement on a proposed appropriation level but - again - that the use of the common-pool resource is more efficient if a proposal is adopted by voting than if group members decide individually.

In contrast, our paper focuses on the impact of endogenously implemented institutions in contrast to their exogenous implementation and is therefore more closely related to the contributions by Sutter et al. (2010) and Balafoutas et al. (2013). Sutter et al. (2010) use a public goods game where subjects can decide whether to participate in a voluntary contribution mechanism in combination with a rewarding or punishment option or to play a standard VCM. They find that the endogenous choice of an institutional setting enhances cooperation compared to the situation with exogenously given mechanisms. The study most closely related to our experiment is the one by Balafoutas et al. (2013). They investigate the effect of endogenously implemented institutional settings with the opportunity to redistribute the benefits from investing into a public good across all group members. Each period of the experiment consists of two stages: A collective-choice and a contribution stage. In the collective-choice stage, participants determine the redistribution factor to be implemented in the contribution stage. The redistribution factor allocates the benefits from investing into the public good across the group members and varies between equal payoffs for all group members and payoffs proportional to subjects' individual contributions to the public good. Subjects specify the value of the redistribution factor they favor at the beginning of each period and the median value among all group members is selected by the experimenter and implemented in the contribution stage. They find that subjects' preferences for the redistribution factor depend on their initial endowment. Subjects with a high and middle initial endowment mostly favor redistribution factors associated with payoffs proportional to individual contributions to the public good. In contrast, subjects with a low initial endowment prefer redistribution factors associated with equal payoffs from investing into the public good. Finally, by comparing exogenously and endogenously implemented redistribution factors Balafoutas et al. (2013) find weak evidence that the opportunity to choose the redistribution factor by themselves makes subjects more cooperative compared to a situation with the same redistribution factor implemented exogenously. Similarly, Dal Bó et al. (2013) show that the effect of an institution on the level of cooperation in a prisoner dilemma is larger when the appropriate mechanisms are chosen democratically. Furthermore, they report this effect to be due to a selection and an endogeneity effect. While players who vote for a certain institution 
may differ from those who are opposed to it (selection effect), the endogeneity effect disentangles potential differences in decision behavior between endogenously or exogenously determined settings.

Contrary to the consistent findings that the democratic implementation of institutions itself enhances cooperation in social dilemma situations, Vollan et al. (2013) show that this effect depends on the societal norms of subjects. Based on existing findings from western democratic countries (Tyran and Feld 2006), they conduct a comparable experiment in China. Their public goods game consists of two scenarios: One in which subjects can decide democratically over the implementation of a sanctioning mechanism, and one in which the contribution schemes are implemented exogenously. In contrast to previous results from Tyran and Feld (2006), they find that an endogenously implemented sanctioning mechanism decreases cooperation compared to the situation which the mechanism implemented exogenously. They argue that these differences can be explained by different societal norms with regard to obeying to authorities in China and western democratic countries.

We extend the existing literature by endogenizing the decision on how to share the burden among sovereign and heterogeneous agents in a public goods game and contribute to the following research questions: Which rules regarding how to share the benefits and costs from providing public goods do agents prefer; whether or not heterogeneous agents are able to agree upon a common burden sharing rule; and whether the endogenous choice of a burden sharing rule has an impact on the willingness to cooperate.

\section{Experimental Design and Theoretical Predictions}

Participants in the Multi-Phase Game play a collective-choice phase and contribution phase. In the collective-choice phase, subjects themselves determine the contribution scheme to be implemented in the contribution phase. Participants in Single-Phase Game play only the contribution phase whereby the different contribution schemes are exogenously given. In the Multi-Phase Game, we first test the effect of the endogenous institutional choice by comparing contributions to the public good of groups which adopt a contribution scheme with contributions from groups which fail to agree upon a scheme. Secondly, we investigate if the endogenous choice impacts cooperation levels by comparing the results from the Multi-Phase Game with the exogenously implemented counterparts from the Single-Phase Game. ${ }^{3}$

\footnotetext{
${ }^{3}$ Details of the experimental design are summarized in Table A.1.
} 


\subsection{Single-Phase Game}

In the Single-Phase Game, subjects only play the contribution phase of the experiment. The contribution phase contains one of four different allocation rules: Three rule-based contribution schemes (eqcont, eqpay and propcont) and the standard voluntary contribution mechanism (VCM).

The payoff to player $i, \pi_{i}$, in all contribution schemes is determined by a linear public goods game and given by

$$
\pi_{i}=e_{i}-q_{i}+b Q
$$

where $e_{i}$ marks the initial endowment, $q_{i}$ the individual contribution to the public good, $b$ the marginal per capita return from the public good for player $i$ and $Q=\sum_{j=1}^{n} q_{j}$ the aggregated provision level. Players differ with respect to their initial endowment. The initial endowment as well as contribution levels and payoffs are expressed in LabDollar (LD). Each group of three players consists of one "low-type" player (type20) with an initial endowment of $e_{\text {type } 20}=20$, one "middle-type" player (type30) with $e_{\text {typezo }}=30$ and one "high-type" player (type40) with $e_{\text {type } 40}=40$. Thus, there is a total group endowment of $E=$ $\sum_{i=1}^{n} e_{i}=90$. Finally, we assume the marginal benefit from the public good to be $b=0.6$ and the marginal costs for investing into the public good to be equal to one.

In the baseline $V C M$, agents simultaneously decide on their individual contributions to the public good, $q_{i}$. Since their marginal costs from investing into the public good exceed their individual benefits, standard theory predicts full free-riding and zero contributions for all players and individual payoffs of $\pi_{i}=e_{i}$.

Our rule-based contribution schemes consist of two stages: In the first stage, the minimum stage, all players simultaneously suggest a minimum group provision level $Q_{i}^{\text {min }} \in[0,90]$. ${ }^{4}$ The smallest suggested proposal then determines the lower bound for aggregated contributions in the second stage. Therefore, $Q^{\min }=\min _{j \in S} Q_{j}^{\min }$ where $S$ is the set of players in a group. In the individual contribution stage, the minimum individual contribution level, $q_{i}^{\min }$, is derived from the binding group minimum provision level, $Q^{\min }$, according to a specific burden sharing rule, i.e., $q_{i}^{\min }\left(Q^{\text {min }}\right)$, whereby subjects have to contribute at least the minimum contribution level $q^{\text {min }}$, i.e., $q_{i} \geq q_{i}^{\text {min }}$. Players can, however, go beyond these minimum requirements and voluntarily contribute more. We cover three different rule-based contribution schemes:

\footnotetext{
${ }^{4}$ In our experiment, for $Q_{i}^{\min }$ integer multiples of three are required.
} 


\section{Equal-Contribution Scheme (eqcont)}

The egalitarian scheme requires equal contribution (eqcont) from all players to the public good such that individual minimum contribution levels are for sufficient low group provision levels given by

$$
q_{i}^{\min }=\frac{1}{n} Q^{\min }
$$

Thus, the binding minimum proposal, $Q^{\text {min }}$, is as far as possible equally distributed across all group members. Note that in burden sharing schemes, in the second stage of the contribution phase it is obligatory that the desired aggregated provision level $Q^{\min }$ is provided by the group and that $q_{i}^{\text {min }} \in\left[0, e_{i}\right]$. It follows that eqcont may require higher contributions from subjects with high endowments if the equal contribution rule would require contributions from low-type players that exceed their initial endowment. More formally, contributions of all group members are equal and given by $q_{i}^{\min }=\frac{1}{n} Q^{\min }$ if $Q^{\min } \in[0,60]$. If $\left.\left.Q^{\text {min }} \in\right] 60,80\right]$, minimum contributions of type20 players are $q_{\text {type } 20}^{\min }=e_{\text {type } 20}$ and therefore lower than those of type30 and type 40 players, $q_{\text {type } 30}^{\min }=q_{\text {type } 40}^{\min }=\frac{1}{n-1}\left(Q^{\min }-e_{\text {type } 20}\right)$. If $Q^{\min } \in$ ]80,90], minimum contributions are given by $q_{\text {type } 20}^{\min }=e_{\text {type } 20}<q_{\text {type30 }}^{\min }=e_{\text {type } 30}<$ $q_{\text {type } 40}^{\min }=\frac{1}{n-2}\left(Q^{\text {min }}-e_{\text {type } 20}-e_{\text {type30 }}\right)$.

In the eqcont scheme, type20 and type30 players have a weakly dominant strategy to suggest $Q_{i}^{\text {min }}=E$. For them the marginal benefit from increasing the groups' binding minimum contribution level exceed its costs for all $Q_{i}^{\min } \in[0, E]$. Choosing $Q_{i}^{\min }<E$ would either reduce their own payoff (i) if they set the binding minimum or (ii) would not change the payoff if their minimum proposal is not pivotal. Following this intuition, type40 players have a weakly dominant strategy to choose $Q_{i}^{\min }=78$, since their marginal payoff is positive only if $Q^{\text {min }} \leq 80.5$ Since the smallest proposal is binding, a subgame perfect equilibrium in weakly dominant strategies is characterized by $Q^{\min }=78$ with $q_{\text {type20 }}^{\min }=20$ and $q_{\text {type } 30}^{\min }=$ $q_{\text {type } 40}^{\min }=29$ which results in payoffs of $\pi_{\text {type } 20}=46.8, \pi_{\text {type } 30}=47.8$ and $\pi_{\text {type } 40}=$ 57.8, given that $q_{i}=q_{i}^{\min }$ (see Table A.2, Figure B.1, and Appendix C for the analytical solution).

\footnotetext{
${ }^{5}$ Mathematically, the weakly dominant strategy of type 40 players is to choose $Q_{i}^{\min }=80$, but the minimum group provision level has to be an integer multiple of three. Therefore, the weakly dominant strategy is to choose the greatest integer multiple of three below 80 .
} 


\section{Equal-Payoff Scheme (eqpay)}

If a rule-based contribution scheme aims at reaching equality in payoffs (eqpay) among all group members and if all types of players have different initial endowments but the same marginal benefits from the public good, equating $\pi_{\text {type } 20}=\pi_{\text {type } 30}=\pi_{\text {type } 40}$ and solving for $q_{i}^{\text {min }}$ implies for sufficient high group provision levels that

$$
q_{i}^{\min }=e_{i}-\frac{1}{n}\left(E-Q^{\min }\right) .
$$

Note that in the eqpay scheme, the desired aggregated provision level $Q^{\text {min }}$ may not entirely allow for payoff equalization since we do not allow for direct redistribution of initial endowments between group members and $q_{i}^{\min } \in\left[0, e_{i}\right]$. This implies that payoff equalization among all group members could be achieved if $Q^{\min } \in[30,90]$. If $Q^{\min }<30$ payoffs are as far as possible equalized. More formally, if $\left.Q^{\min } \in\right] 9,30\left[, q_{\text {type20 }}^{\min }=0\right.$ and $q_{\text {type } 30}^{\min }=q_{\text {type } 40}^{\min }=e_{j}-\frac{1}{n-1}\left(e_{\text {type } 40}+e_{\text {type30 }}-Q^{\min }\right) . \quad$ If $\quad Q^{\min } \in[0,9], \quad q_{\text {type20 }}^{\min }=$ $q_{\text {type } 30}^{\min }=0$ and $q_{\text {type } 40}^{\min }=Q^{\min }$. For instance, if $Q^{\min }=24$, this scheme would require $q_{\text {type } 20}^{\min }=0, q_{\text {type } 30}^{\min }=7$ and $q_{\text {type40 }}^{\min }=17$ but nevertheless due to the endowment heterogeneity payoff equality is not reached. If $q_{i}=q_{i}^{\text {min }}$ individual payoffs are given by $\pi_{\text {type } 20}=34.4, \pi_{\text {type } 30}=\pi_{\text {type } 40}=37.4$.

In the eqpay scheme, we expect all players to suggest full contribution levels, i.e., $Q_{i}^{\text {min }}=E$ (see Table A.2, Figure B.1, and Appendix C for the analytical solution). type20 and type30 players have a weakly dominant strategy to suggest $Q_{i}^{\min }=E$. By anticipating the weakly dominant strategy of type20 and type30 players, also type 40 players maximize their payoff by suggesting $Q_{i}^{\min }=E$. This allocation would lead to individual minimum contribution of $q_{\text {type } 20}^{\min }=e_{\text {type } 20}, q_{\text {type } 30}^{\min }=e_{\text {type } 30}$ and $q_{\text {type } 40}^{\min }=e_{\text {type } 40}$ and given that $q_{i}=q_{i}^{\min }$ equal payoffs for all group members of $\pi_{\text {type } 20}=\pi_{\text {type } 30}=\pi_{\text {type } 40}=54$. $^{6}$

\section{Proportional-Contribution Scheme (propcont)}

If a rule-based contribution scheme requires individual contributions to be proportional to players’ initial endowment (propcont), individual minimum contribution levels are given by

$$
q_{i}^{\min }=\frac{e_{i}}{E} Q^{\min }
$$

\footnotetext{
${ }^{6}$ type40 players' payoff function is not monotonic increasing in $Q^{\mathrm{min}}$. Therefore, depending on their beliefs about the other players' proposals, type 40 players' best response is to propose $Q_{\text {type } 40}^{\min }=0$ or $Q_{t y p e 40}^{\min }=E$. We assume type 40 players to anticipate the weakly dominant strategies of their group members and since then their payoff is maximized by $Q_{t y p e 40}^{\min }=E$, we expect type 40 players to propose full contributions.
} 
Thus, the binding minimum proposal, $Q^{\text {min }}$, is distributed across group members according to their fraction on the total endowment, that implies, that individual minimum contributions increase with players' initial endowment. In this case, all players have a weakly dominant strategy to suggest full contribution levels, i.e., $Q^{\min }=E$, since their marginal benefit from increasing the group's minimum contribution level exceeds its costs all $Q^{\text {min }} \in[0, E]$. This allocation is a subgame perfect equilibrium in weakly dominant strategies and would lead to individual minimum contribution of $q_{\text {type } 20}^{\min }=e_{\text {type20 }}, q_{\text {type30 }}^{\min }=e_{\text {type30 }}$ and $q_{\text {type } 40}^{\min }=$ $e_{\text {type } 40}$ and given that $q_{i}=q_{i}^{\min }$ to payoffs of $\pi_{\text {type } 20}=\pi_{\text {type } 30}=\pi_{\text {type } 40}=54$ (see Table A.2, Figure B.1, and Appendix C for the analytical solution).

Thus, assuming that subjects behave according to standard theory and play their minimum contribution level, i.e., $q_{i}=q_{i}^{\text {min }}$, we can derive the following hypothesis regarding groups' contribution levels

\section{H1: Aggregate Contribution Level to the Public Good}

$$
Q_{\text {eqpay }}=Q_{\text {propcont }}>Q_{\text {eqcont }}>Q_{V C M}=0 .
$$

The theoretical predictions for all rule-based contribution schemes are summarized in Table A.2 and graphically illustrated in Figure B.1.

\subsection{Multi-Phase Game}

In the first stage of the Multi-Phase Game, the collective-choice phase, subjects anonymously vote for one of the proposed rule-based contribution schemes (eqcont, eqpay and propcont). We investigate two different voting mechanisms, majority and unanimity rule voting, each with three voting rounds. After each voting round, all group members are informed about the voting behavior of their group members and whether an agreement is reached. To be adopted, a rule-based contribution scheme has to receive 2 out of 3 votes (majority rule voting) or 3 out of 3 votes (unanimity rule voting). If the sufficient majority is obtained, the corresponding burden sharing rule is implemented thereafter in the contribution phase. If a majority cannot be obtained after the third voting round, participants play the VCM.

According to standard preferences, we expect type20 and type30 players to vote more frequently for the eqpay and propcont schemes compared to the eqcont scheme since their expected payoffs are comparatively high in those schemes (see Table A.2 and Figure B.1). In contrast, for type40 players the expected payoff is the highest in the eqcont scheme. 
Therefore, we expect type 40 players to vote more frequently on the eqcont scheme compared to the eqpay and propcont scheme. Since the VCM is expected to be payoff-dominated by all rule-based contribution schemes, we expect participants to jointly agree upon a rule-based contribution scheme. Furthermore, we expect that an agreement is more easily reached under majority than unanimity rule voting and groups to agree more frequently on the eqpay and propcont schemes compared to eqcont.

By relaxing the assumptions of standard preferences and allowing for other-regarding preferences, it can be expected that also type40 players vote for the eqpay scheme. One prominent theory of other-regarding preferences which in addition allows simple utility calculations is the inequality version model by Fehr and Schmidt (1999, F\&S). They formalize the idea of inequality aversion by adding a disutility from disadvantageous inequality (weighted by parameter $\alpha_{i}$ ) and a disutility from advantageous inequality (weighted by parameter $\beta_{i}$ ) to a standard linear utility function. F\&S themselves present mean values $\bar{\beta}=0.315$ and $\bar{\alpha}=0.85$ for the inequality aversion parameters which they derive from individual behavior in ultimatum games. Blanco et al. (2011) use modified ultimatum and dictator games to obtain similar inequality aversion parameters. In their distribution ( $n=61$ ), $56 \%$ of all subjects can be characterized by $\beta_{i}>0.5$ and $33 \%$ have $\beta_{i}>0.67$. In our case, for $\beta_{i}>0.67$ a type 40 player prefers eqpay to the alternative burden sharing rules. For type30 and type40 players with $\beta_{i} \geq 0$ and $\alpha_{i} \geq 0$, eqpay at least weakly dominates the alternatives. ${ }^{7}$ Therefore, we can summarize our expectations in the following hypothesis:

\section{H2: Voting in the Collective-Choice Phase of the Multi-Phase Game}

a) According to standard preferences type20 and type30 participants vote more frequently for the eqpay and propcont schemes compared to the eqcont scheme. In contrast, type 40 players vote more frequently for the eqcont scheme.

b) Participants agree upon a rule-based contribution scheme. They agree more frequently on the eqpay or propcont scheme compared to the eqcont scheme and an agreement is more frequently observed under majority compared to unanimity rule voting.

c) By allowing for other-regarding preferences it can be expected that also type 40 players vote more frequently for the eqpay scheme compared to the eqcont and propcont schemes. 
Furthermore, in our experiment collective-choice itself becomes a treatment variable to distinguish whether a contribution scheme is implemented exogenously by the experimenter or endogenously by voting of the subjects. To distinguish between exogenously and endogenously implemented contribution schemes, we add the prefix ex- (i.e., ex-VCM, exeqcont, ex-eqpay and ex-propcont) for the exogenous case and the prefix end- (i.e., end-VCM, end-eqcont, end-eqpay and end-propcont) for the endogenous case. As standard theory is silent regarding the way how the institution has been developed, we do not expect any differences in behavior in exogenously and endogenously implemented contribution schemes. Thus, we can state the hypothesis:

\section{H3: Endogenous and Exogenous Choice of Contribution Schemes}

$$
Q_{\text {ex-i }}=Q_{\text {end-i }} \text { whereby } i=V C M, \text { eqcont, eqpay, propcont }
$$

Nevertheless, previous studies have shown that the choice of an institutional setting itself may have a positive effect on the level of cooperation in social dilemma situations. ${ }^{8}$ Therefore, we have reasonable doubts with respect to the validity of $\mathrm{H3}$ and could expect that the opportunity to choose a rule-based contribution scheme by themselves makes subjects more cooperative.

\subsection{Laboratory Protocol}

The experiment was run in July 2013 at the MaxLab laboratory of the University of Magdeburg in Germany. We used ORSEE (Greiner 2004) for recruiting participants and zTree software (Fischbacher 2007) for programming. We recruited 363 students from different disciplines. Each student took part in one of 16 sessions with between 21 and 24 subjects. On average, a session lasted about 60 minutes. In each session we randomly created up to eight groups of three players with different initial endowments, each group consists of one type20, type30 and type40 player. Each player remained the same type and in the same group throughout the whole experiment (partner matching). During the collective-choice phase, subjects received information on players' type and the outcome of the election within their group. During the contribution stage, information on individual contributions to the public good, payoffs and corresponding average values within the group was transmitted via screen. Participants were not aware of their exact partners and no direct communication between participants was allowed.

\footnotetext{
${ }^{8}$ See Section 2 for a brief review of the related literature.
} 
At the beginning, participants received a set of experimental instructions which included written descriptions, numerical examples, and control questions. Furthermore, participants could make use of a simulator on their screen to verify the numerical examples, to answer control questions and to simulate different contribution decisions. ${ }^{9}$ A session of the SinglePhase Game consists of 12 rounds of the public goods game, the first two being practice rounds. Additionally, in the Multi-Phase Game the collective-choice phase with a maximum of three voting rounds was added. At the end of each session, one non-practice round of the public goods game was randomly chosen to determine individual earnings. The exchange rate between Euro and LD was 1:3. On average, participants earned 14.70 Euro. No additional show-up fee was paid.

\section{Experimental Results}

\subsection{Collective-Choice Phase}

We begin our discussion by analyzing participants' voting behavior in the collective-choice phase of the Multi-Phase Game. Our main findings are summarized in Figure 1.

\section{Figure 1: Individually Chosen Rule-Based Contribution Schemes}

by types

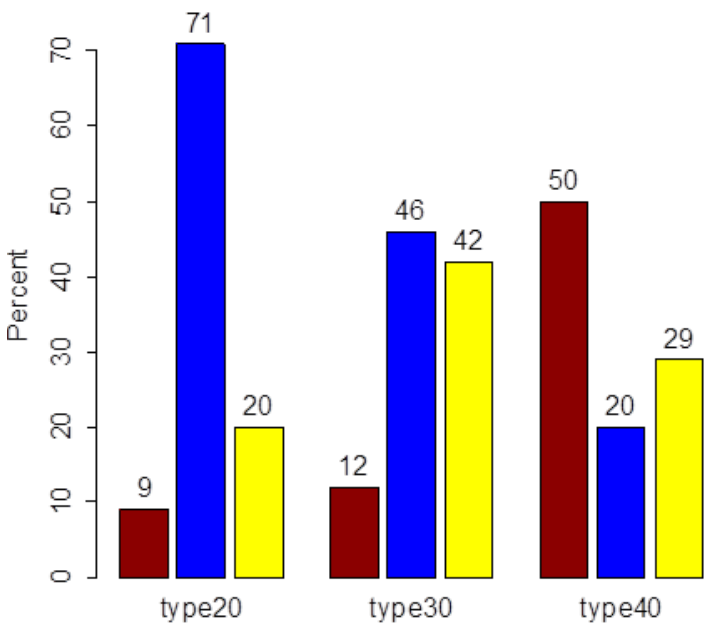

Note: Individually chosen rule-based contribution scheme in all three rounds of the collective-choice phase of the Multi-Phase Game by types. by rounds

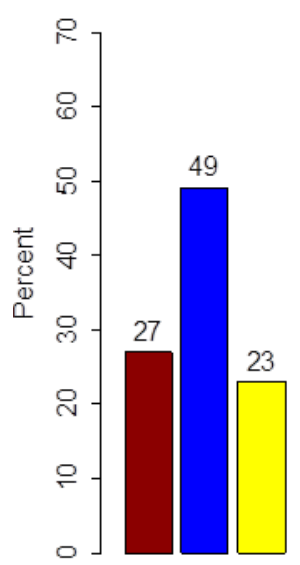

Round 1

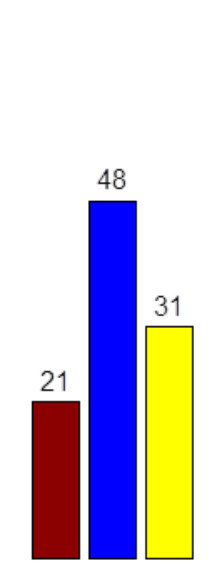

Round 2
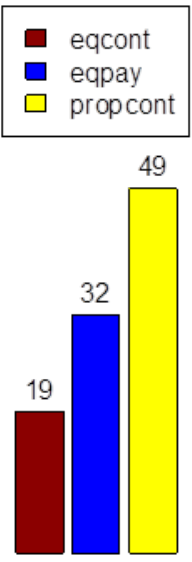

Round 3

$\begin{array}{lccc}\text { votes } & 180 & 117 & 72 \\ \text { groups } & 21 & 15 & 10\end{array}$

Notes: Individually chosen rule-based contribution scheme of all players in the collective-choice phase of Multi-Phase Game by rounds. Votes: absolute number of votes per round. Groups: absolute number of groups which successfully coordinate.

\footnotetext{
${ }^{9}$ We provide an example of instructions and screenshots in the Appendix E and F.
} 


\section{Observation 1: Individually Chosen Rule-Based Contribution Scheme}

The rule-based contribution scheme subjects prefer depends on their initial wealth position in the group: The higher their initial endowment, the less (more) frequently they vote for the eqpay (eqcont) scheme. The propcont scheme is most frequently chosen by middle endowed players.

In line with our theoretical prediction, we find preferences for the different allocation rules to depend on subjects' initial endowment: The frequency players vote for the eqpay scheme is decreasing in the initial endowment ( $\mathrm{p}<0.01$, Binomial Test, Table A.3). type20 players vote in $71 \%$ of all cases for the eqpay scheme, type30 players in $46 \%$ and type 40 players in $20 \%$, respectively. Furthermore, type 40 players vote more frequently for the eqcont scheme than the other group members (type40: 50\%, type30: 12\%, type20: $9 \%)(\mathrm{p}<0.01$, Binomial Test, Table A.3). The propcont scheme is most frequently chosen by type30 players (type40: 29\%, type30: 42\%, type20: 20\%) ( $<<0.05$, Binomial Test, Table A.3).

Table 1: Implemented Contribution Schemes in the Multi-Phase Game

\begin{tabular}{|c|c|c|c|}
\hline & Majority rule voting & Unanimity rule voting & Total \\
\hline \multirow[t]{2}{*}{$V C M$} & 1 & 13 & 14 \\
\hline & (3.5) & (41.9) & $(23.3)$ \\
\hline \multirow[t]{2}{*}{ eqcont } & 3 & 2 & 5 \\
\hline & (10.3) & $(6.5)$ & (8.3) \\
\hline \multirow[t]{2}{*}{ eqpay } & 18 & 10 & 28 \\
\hline & $(62.1)$ & (32.3) & $(46.7)$ \\
\hline \multirow[t]{2}{*}{ propcont } & 7 & 6 & 13 \\
\hline & $(24.1)$ & $(19.4)$ & $(21.7)$ \\
\hline Total & 29 & 31 & 60 \\
\hline
\end{tabular}

Notes: Contribution schemes chosen by a group serves as one observation. Percentage in parentheses.

\section{Observation 2: Implemented Contribution Scheme in the Multi-Phase Game}

The majority of all groups managed to agree upon a common burden sharing rule. Furthermore, groups agree more frequently under majority rule voting compared to unanimity rule voting and eqpay is the most frequently chosen rule-based contribution scheme.

Turning our analysis towards a potential agreement, our experimental data suggest that with $77 \%$ the majority of all groups exploit the potential efficiency gains the rule-based contribution schemes offer and agree upon a common burden sharing rule. We find a common agreement is more easily obtained under majority (97\%) than under unanimity rule voting 
(58\%) $\left(\mathrm{p}<0.01, \chi^{2}\right.$-test and Fisher's exact test). This could be due to heterogeneous preferences among the individual group members. Furthermore, we find that eqpay is the most frequently chosen burden sharing rule ( $<<0.01$, Binomial Test, Table A.4). $47 \%$ of all groups adopt the eqpay scheme to be implemented in the contribution phase. The propcont scheme is chosen by $22 \%$ of all groups and eqcont by $8 \%$ of all groups (see Table 1 ).

While at first glance the standard theory of rational and selfish behavior performs quite well regarding subjects' voting behavior, it remains noteworthy, however, that the eqpay scheme is, with $62 \%$ under majority voting and still with 32\% under unanimity voting, the most frequently chosen contribution scheme (see Table 1). This observation cannot be explained by standard theory. One plausible explanation is that a significant fraction of subjects is motivated by other-regarding preferences. In particular, the behavior of type40 players who vote for eqpay can be explained very plausibly by inequality aversion (e.g., Fehr and Schmidt 1999). By assuming a sufficiently high level of disutility from advantageous inequality, type 40 players prefer the eqpay to the eqcont and propcont schemes. Thus, inequality aversion may explain the observed voting behavior. ${ }^{10}$

\section{Observation 3: Chosen Burden Sharing Rule by Rounds}

Almost half of the groups which agree upon a common burden sharing rule already reach an agreement in the first voting round. Participants of groups who do not reach consensus in the first place change their voting behavior over the rounds: They vote less (more) frequently for the eqpay (propcont) scheme.

We find that almost half of all groups (21 groups, 45\%) that manage to agree upon a common rule-based contribution scheme, already reach consensus in the first round of the collectivechoice phase (see Figure 1). In the second round, further 15 groups agree on an allocation scheme and finally, further 10 groups in the last voting round. Next, over the periods, participants vote less frequently for the eqpay scheme and more frequently for the propcont scheme ( $<0.05$, Binomial Test, Table A.5). We do not find any significant changes regarding the voting behavior on the eqcont scheme. Finally, 14 out of the 60 groups in the Multi-Phase Game fail to agree upon a common burden sharing rule. This could be due to divergent preferences or, since we do not allow for a direct communication etc., a coordination problem. This means that group members are principally willing to agree upon a common rule but fail to coordinate. By analyzing group member's individual voting behavior over the three rounds

\footnotetext{
${ }^{10}$ See Appendix D for a detailed description and a graphical illustration.
} 
of the collective-choice phase we conclude that most groups who fail to agree upon a common burden sharing rule do so because of the missing willingness to cooperate and to compromise. We find that in 11 out of the 14 groups who failed to agree upon a common burden sharing rule at least one group member is not willing to give up this initially chosen allocation rule over the rounds of the voting stage and insists on the initially chosen burden sharing rule. This inflexible position decreases the likelihood of reaching consensus, as this would require that the other group members change their voting behavior.

\subsection{Contribution Phase}

\subsubsection{Contributions to the Public Good}

In this section, we analyze subjects' contributions to the public good both in the Single- and the Multi-Phase Game. Table A.6 reports average group contribution levels for each endogenously and exogenously implemented contribution scheme across all periods excluding trial periods. ${ }^{11}$

At first we investigate average contributions to the public good in the endogenous case. The corresponding results are depicted in Figure 2. In line with our theoretical considerations, we find contributions to the public good to be higher in case of reaching an agreement upon a common distribution rule than in case of no rule is adopted ( $<<0.10$, MW-U test, Table A.7). Averaged over all periods and agents, contributions are lowest if subjects could not agree upon a common rule-based contribution scheme (8.1) and highest if participants agreed upon the eqpay scheme (26.3). Average contributions in the endogenously implemented eqcont and propcont schemes are 20.3 and 22.5 respectively (see Table A.6). Our regression results (see Table A.13 Model 1) confirm these observations. In Model 1, we only consider observations from participants in the Multi-Phase Game and find that the level of contributions to the public good is higher if groups adopt a rule-based contribution scheme in the collectivechoice phase. Groups who adopted the eqcont, eqpay or propcont schemes contribute significantly more than groups who failed to agree upon a common burden sharing rule and therefore have to play the VCM.

\footnotetext{
${ }^{11}$ We further provide results of nonparametric Mann-Whitney U (MW-U) tests regarding the contributions in Table A.7. Results of nonparametric Jonckheere-Terpstra test on time trends in contributions are given in Table A.8. Further statistical evidence is given by a series of random effects regression models; see Table A.13. A description of all dependent and independent variables entering our regression models is given in Table A.12.
} 
Figure 2: Average Contributions per Group in Endogenously and Exogenously Implemented Contribution Schemes
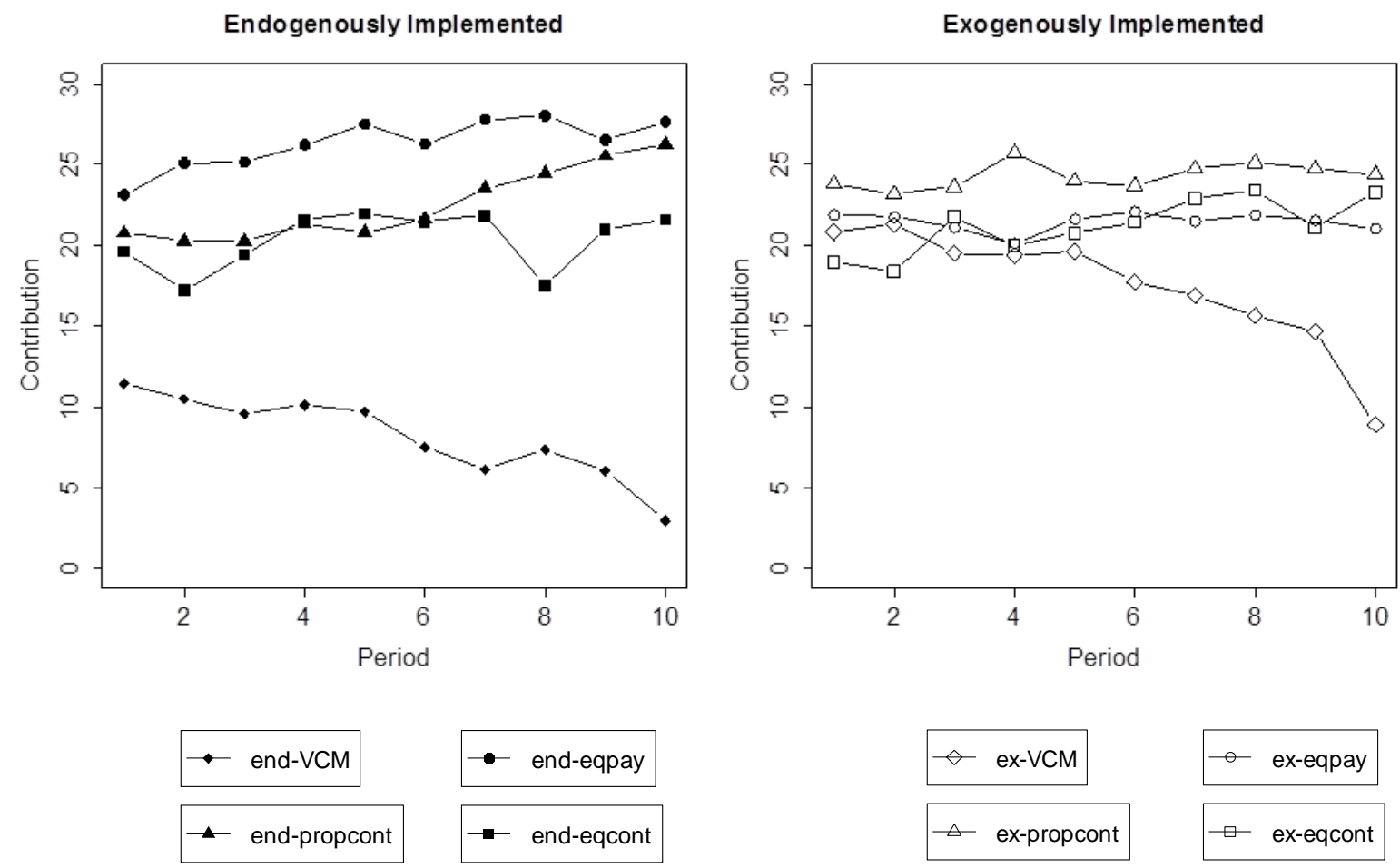

Notes: Average group contributions in each period (excluding the trial periods) of the game serve as one observation. On the left (right): contributions in the endogenously (exogenously) implemented contribution schemes.

\section{Observation 4: Contributions in Endogenously Implemented Contribution Schemes}

Average contributions are higher in case a rule-based contribution scheme is successfully endogenously adopted than in case a sufficient majority is failed and contribution decisions are made in the VCM. The downward trend in contributions disappears if a rule-based contribution scheme is adopted.

In addition, for eqpay, we find that contributions are higher in case the scheme is implemented by unanimity (28.8) in contrast to majority rule voting (24.4) (p<0.10, MW-U test, Table A.9 and Figure B.2 left side). All in all, 28 groups choose the eqpay scheme and among those 12 groups come to this decision unanimously. ${ }^{12}$ Noteworthy is that in 12 out of the remaining 16 groups, in which the eqpay scheme is implemented by majority, type40 players are overruled. Focusing on these observations, we find that overruled type40 players are comparatively less cooperative (see Figure B.2 right side). type40 players contribute 33.1

\footnotetext{
${ }^{12}$ Note that two groups agreed under majority rule voting per unanimity on the eqpay scheme.
} 
if they were overruled in contrast to 37.8 if they voted for eqpay. ${ }^{13}$ This observation is statistically significant for the last period of the contribution phase. Here, overruled type40 players contribute on average 34.9 and not-overruled type40 players $40.0(\mathrm{p}<0.01$, MW-U test).

Finally, we find that the agreement on a common rule-based contribution scheme counteracts downward trends in contributions over periods. Contributions of groups, who have to play the $V C M$, decline over the periods ( $\mathrm{p}<0.01$, Jonckheere-Terpstra test, Table A.8). In all rule-based contribution schemes, there is no evidence for a negative time trend. In the endogenously implemented eqpay and propcont schemes, contributions even significantly increase over the periods ( $<<0.1$, Jonckheere-Terpstra test, Table A.8).

In the next paragraph, we turn our analysis towards the different contribution schemes given that they are implemented exogenously.

\section{Observation 5: Contributions in Exogenously Implemented Contribution Schemes}

Given that the contribution schemes are implemented exogenously, the level of cooperation is higher in rule-based contribution schemes compared to the VCM. Again, rule-based contribution schemes counteract the downward trend in contributions.

Comparable to the results of the Multi-Phase Game we, find that the average contributions are higher in rule-based contribution schemes than in the VCM (see Table A.6). Averaged over all periods and agents, contributions in the propcont scheme are with 24.3 the highest and significantly higher than those in the VCM (17.5) ( $<<0.05$, MW-U test, Table A.7). In the eqpay and eqcont schemes average contributions are 21.5 and 21.2, respectively, but not statistically significant different from average contributions in the VCM. In our regression analysis (see Table A.13 Model 2), we find that the different exogenously implemented contribution schemes (eqpay, eqcont and propcont) have a significantly positive impact on subjects' contributions to the public good compared to the exogenous VCM: the eqpay scheme at a significance level of $1 \%$, the other two rule-based contribution schemes at the $10 \%$-level. Finally, we find that also the exogenously given schemes prevent contributions from declining. Given that the VCM is exogenously implemented, average contributions significantly decline over periods ( $\mathrm{p}<0.01$, Jonckheere-Terpstra test, Table A.8). In contrast, there is no evidence that the average contributions decline in the exogenous implemented

\footnotetext{
${ }^{13}$ We could not observe this behavior in the eqcont and propcont scheme. One reason could be the small number of groups that agreed in the collective-choice phase of the Multi-Phase Game on the eqcont or propcont scheme.
} 
rule-based contribution schemes and in the eqcont scheme average contributions increase significantly over the periods ( $\mathrm{p}<0.05$, Jonckheere-Terpstra test, Table A.8).

Next, we turn our analysis towards the comparison of contributions to the public good in endogenously chosen contribution schemes and their exogenously implemented counterparts.

\section{Observation 6: Contributions in Endogenously and Exogenously Implemented Contribution Schemes}

a) Considering the eqpay scheme, contributions are higher if this scheme is implemented by voting compared to if it is implemented exogenously.

b) Considering the VCM, subjects who could not manage to agree upon a common rulebased contribution scheme and, hence, choose to play the VCM contribute less compared to participants in the exogenously implemented VCM.

We find that providing the opportunity to reach an agreement by voting has an effect on the level of contributions to the public good. If participants manage to agree upon the eqpay scheme, contributions are higher compared to the exogenous implemented case $(p<0.05, M W$ U test, Table A.7). Averaged over all periods and agents, contributions in the exogenously implemented eqpay scheme are 21.5, if, however, the eqpay scheme is implemented endogenously average contributions are 26.4 (see Table A.6). In contrast, we find that average contributions are lower if participants do not manage to agree upon a common rule-based contribution scheme and, therefore, have to play the VCM compared to the situation in which the VCM is implemented exogenously ( $\mathrm{p}<0.05$, MW-U test, Table A.7). Contributions in the exogenously implemented $V C M$ are 17.5 and 8.1 if participants failed to agree upon a common rule-based contribution scheme in the voting stage of the experiment (see Table A.6). These results are confirmed by our regression results (see Table A.13 Model 3-6). In Model 3-6, we only consider observations from the same exogenously or endogenously implemented contribution scheme (VCM, eqcont, eqpay and propcont). We find that contributions are significantly lower in the endogenously compared to the exogenously implemented VCM (see Table A.13 Model 3). In contrast, contributions in the endogenously implemented eqpay scheme are significantly higher than in the exogenously implemented eqpay scheme (see Table A.13 Model 5). With respect to the eqcont and propcont schemes, we cannot find an effect of endogenous choice on the level of contributions to the public good. 
It could be argued that the differences in subjects' contributions to the public good between endogenously and exogenously implemented contribution schemes are driven by a sample selection effect à la Dal Bó et al. (2013) and not by an effect of the endogenous choice per se. ${ }^{14}$ By assuming that cooperative subjects are rather willing to give up their initial position in the collective-choice phase of the experiment in order to increase the likelihood that their group agrees at least on any burden sharing rule in the next voting round, the collectivechoice phase could select cooperative from uncooperative subjects. Therefore, we could expect higher contributions in groups that manage to agree upon a common rule-based contribution scheme at the end of the collective-choice phase compared to participants that ended up in the VCM. We do not deny a possible sample selection effect but following this logic a sample selection effect should be relatively weak if groups already agreed in the first round of the collective-choice phase on a common allocation rule. By considering only groups who agreed already in the first voting round of the collective-choice phase on the eqpay scheme we find that the opportunity to vote has still a positive impact on subjects' contributions to the public good (see Figure B.3). Contributions in groups that agree in the first voting round on the eqpay scheme are with 25.5 significantly higher than in the exogenously implemented eqpay scheme with 21.45 ( $\mathrm{p}<0.01$, MW-U test). Following this reasoning, we argue that our results are at least partly driven by an effect of the endogenous choice and not solely by a sample selection effect.

\subsubsection{Payoffs}

Analyzing participants' contribution behavior indicates rule-based contribution schemes to be effective in increasing contributions to the public good compared to a VCM and that the endogenous choice of a contribution scheme does affect the level of contributions. In the following, we turn our analysis towards payoff comparisons across the different contribution schemes and methods of implementation. Our analysis, thereby, is driven by the question whether there exists a contribution scheme which payoff-dominates all other contribution schemes. A contribution scheme is payoff-dominant if it is not possible to make any type of player better off by choosing another scheme without making at least one player worse off.

Figure 3 summarizes the average payoffs per group over the periods in all exogenously and endogenously implemented contribution schemes. ${ }^{15}$

\footnotetext{
${ }^{14}$ See Section 2 for a brief review of the related literature.

15 Table A.6 reports average group payoffs for each endogenously and exogenously implemented contribution scheme across all periods excluding trial periods. We further provide results of MW-U tests regarding the
} 
Figure 3: Average Payoffs per Group in Endogenously and Exogenously Implemented Contribution Schemes
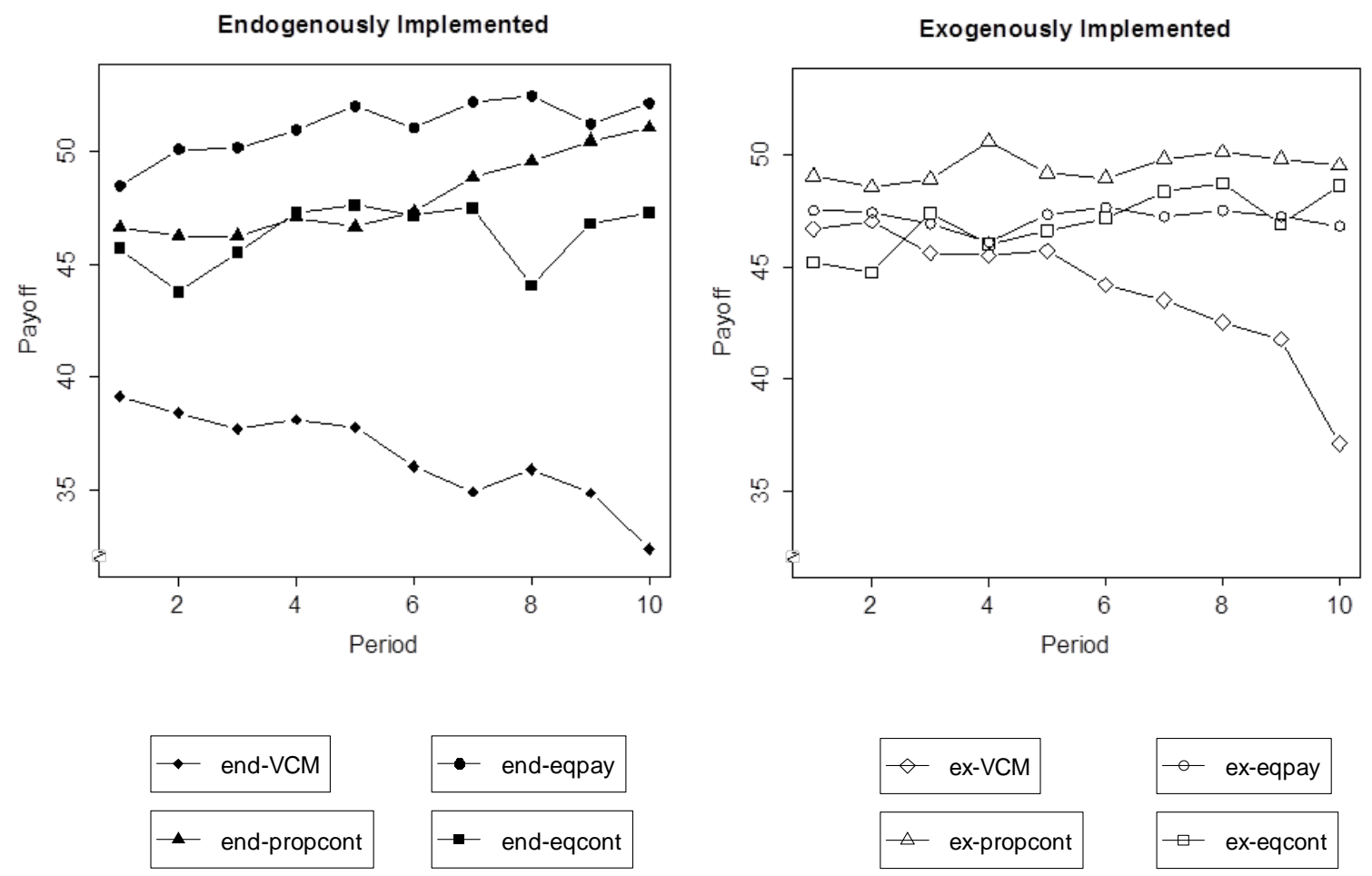

Notes: Average group payoffs in each period (excluding the trial periods) of the game serve as one observation. On the left (right): Payoffs in the endogenously (exogenously) implemented contribution schemes.

As indicated by the contribution behavior, we find compared over all contribution schemes and methods of implementation average profits per group are lowest if group members could not manage to agree upon a common rule-based contribution scheme in the collective-choice phase and therefore have to play the VCM (36.5) and highest if they agree upon the eqpay scheme (51.2) (see Table A.6). More precisely, we find that average payoffs per group are significantly higher in case one of the three rule-based contribution schemes (eqcont, eqpay or propcont) is adopted than in case no scheme gets a sufficient majority $(\mathrm{p}<0.1$, MW-U test, Table A.10, Table A.14 Model 1).

Also in case the contribution schemes are exogenously implemented, payoffs in the rule-based contribution schemes (eqpay, eqcont and propcont) are higher than in the VCM (see Table A.6) but according to a MW-U test only average profits in the propcont scheme (49.4) are significantly different from those in the $\operatorname{VCM}(44.0)(\mathrm{p}<0.05$, MW-U test, Table A.10). According to the results of our regression analysis (see Table A.14 Model 2), all the rule-

payoffs in Table A.10. Results of nonparametric Jonckheere-Terpstra test on time trends in contributions are given in Table A.11. Further statistical evidence is given by a series of random effects regression models; see Table A.14. A description of all dependent and independent variables entering our regression models is given in Table A.12. 
based contribution schemes (eqpay, eqcont and propcont) have a significantly positive impact on participants' payoffs compared to the VCM.

As indicated by our observations on participants' contribution behavior we find that the opportunity to choose a rule-based contribution scheme in an election has an effect on average payoffs. If participants manage to agree upon the eqpay scheme, profits are significantly higher compared to the exogenously implemented eqpay scheme $(\mathrm{p}<0.1, \mathrm{MW}-\mathrm{U}$ test, Table A.10). In contrast, we find that payoffs are significantly lower if participants did not manage to agree upon a common rule-based contribution scheme and therefore have to play the VCM compared to the situation in which the VCM is implemented exogenously $(\mathrm{p}<0.05, \mathrm{MW}-\mathrm{U}$ test, Table A.10). The results of our regression analysis confirm these observations (see Table A.14 Model 3 and 5).

\section{Observation 7: Individual Payoffs in Exogenously and Endogenously Implemented Contribution Schemes}

a) In the exogenous case, the propcont scheme leads to the highest average payoffs per group and at least weakly payoff-dominates the other contribution schemes.

b) In the endogenous case, the eqpay scheme leads to the highest average payoffs per group but is not payoff dominant.

By turning our analysis towards the individual payoffs of the different types of players per group we find that among the exogenously implemented contribution schemes the propcont scheme at least weakly payoff-dominates all other contribution schemes. Compared to the other contribution schemes, type20 and type30 players receive in the propcont scheme their highest payoffs with 47.2 and 49.5 respectively. type 40 players get the highest payoff in the eqcont scheme (53.3) but this is not statistically different from the payoff in the propcont scheme (51.6). Therefore, we conclude that among the exogenously implemented contribution schemes the propcont is at least weakly payoff-dominant. It is not possible to make any type of player better off compared to the propcont scheme without making at least one player worse off.

The situation changes if we turn our analysis towards the endogenously implemented contribution schemes. If the contribution schemes are implemented by voting in the collective-choice phase of the experiment, the eqpay scheme leads to the highest average payoffs but is not payoff-dominant compared to the other contribution schemes. type20 and type30 players receive their highest payoffs in the eqpay scheme with 50.3 and 51.3, respectively. type40 players get their highest payoff in the eqcont scheme with 52.7 and 
therefore significantly more than in the endogenously implemented eqpay scheme with 51.5 $(\mathrm{p}<0.1, \mathrm{MW}-\mathrm{U}$ test $)$.

\subsubsection{Minimum Group Contribution Levels}

While analyzing participants' contribution behavior and the corresponding payoffs we find that rule-based contribution schemes are effective in increasing contributions to the public good which leads to higher payoffs and that it makes a difference how the contribution schemes are implemented. In case of the eqpay scheme, we find that contributions and corresponding payoffs are higher in case the scheme is implemented endogenously compared to the exogenously implemented eqpay scheme.

Considering the latter positive voting effect in more detail we are turning our analysis towards the binding minimum group contribution levels (see Figure 4) and the individual proposals for the minimum group contribution level. As indicated by participants' contribution behavior and the corresponding payoffs we find that the average binding minimum group contribution level is higher in case the eqpay scheme is implemented endogenously (76.6) than in case the same scheme is implemented exogenously (61.4) ( $\mathrm{p}<0.1$, MW-U test, Table A.15). By focusing on groups who failed to coordinate on the full contribution level, we find that type40 players are in most cases the pivotal group members, i.e., those group members whose proposals set the binding minimum group contribution level. Looking at the individual rounds of the contribution phase, we find that in $50.6 \%$ of the endogenously implemented eqpay schemes and in $41.8 \%$ of the exogenously implemented eqpay scheme type 40 players set the binding minimum group contribution level.

More precisely, we find that independent from the method of implementation type 40 players make on average the smallest proposals for the binding minimum group contribution level. In case the eqpay scheme is chosen in the collective-choice phase they propose on average a binding contribution level of 81.2. In case the scheme is implemented exogenously, they propose on average 73.9. type30 players propose on average 83.6 in the endogenous and 77.2 in the exogenous case and type20 players 85.7 and 77.9, respectively. Furthermore, these numbers show that all types of players propose on average more for the binding group contribution level in case the eqpay scheme is implemented endogenously compared to the exogenously implemented eqpay scheme. 
Figure 4: Average Binding Group Minimum Contribution Levels in Endogenously and Exogenously Implemented Contribution Schemes
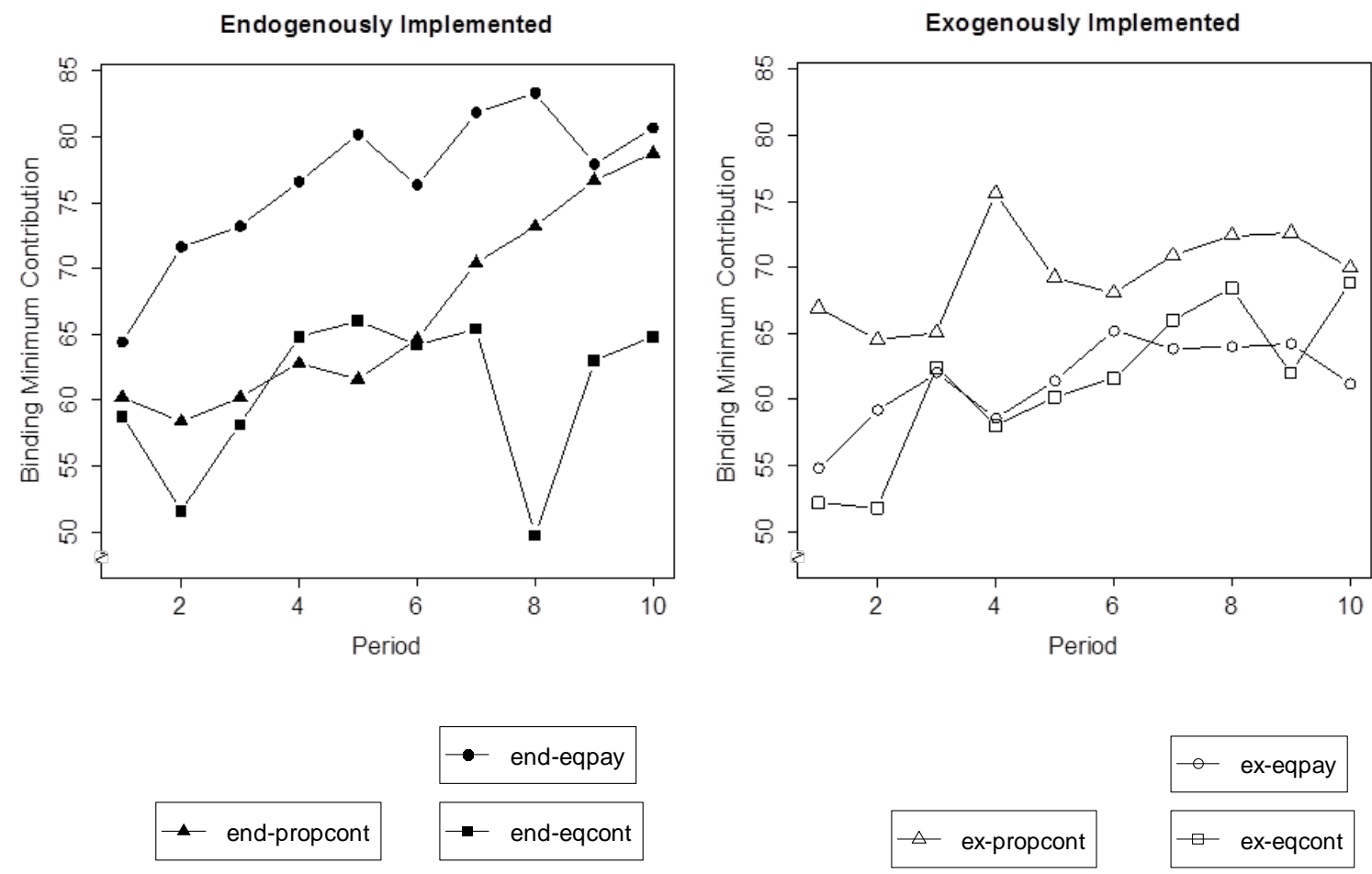

Note: Average binding group minimum contribution levels in each period (excluding the trial periods) of the game serve as one observation. On the left (right): Average binding group minimum contribution level in the endogenously (exogenously) implemented contribution schemes.

\section{Observation 8: Proposals for the Binding Minimum Group Contribution Level}

a) type40 players propose on average the smallest suggestions for the binding minimum group contribution level.

b) All players propose on average more for the binding minimum group contribution level in case the eqpay scheme is implemented endogenously compared to the exogenously implemented eqpay scheme.

c) type40 players have the smallest voluntary contributions beyond the minimum individual contribution levels.

\section{Summary and Concluding Remarks}

In this experiment, we investigate whether the endogenous choice of different rules how to share the costs of providing a public good affects individual contribution behavior. International climate policy might serve as a possible application for this framework. In climate negotiations, delegates try to agree upon an overall greenhouse gas reduction target in 
combination with a burden sharing rule for the distribution of the overall reduction target among the participating countries. A variety of such rules is conceivable and it is a major challenge for the negotiators to agree upon a common rule how to distribute the overall burden among the participating countries. Also in the management of local commons, e.g., irrigation systems, different rules that govern the distribution of costs and benefits of cooperation among group members are subject of discussion. Institutions with congruent rules for distributing the costs and benefits lead to comparatively high cooperation levels, but again - a variety of different rules is conceivable and it remains unclear under what circumstances which rules evolve.

Our major finding is that the endogenous choice of a contribution scheme does affect the level of contributions to the public good. In case of the eqpay scheme, subjects contribute more to the public good if they have selected the scheme themselves instead of a situation where the scheme is implemented exogenously by the experimenter. This effect is even stronger if groups agree by unanimity compared to majority rule on the eqpay scheme. While we observe this positive effect of endogenous institutional choice on contributions to the public good, there is also a negative effect. If group members fail to agree upon a rule-based contribution scheme in the collective-choice phase of the Multi-Phase Game they have to play the VCM. In this endogenous case, contributions are lower than in case the VCM is implemented exogenously. Therefore, we have to reject our initial research hypothesis H3 stating that there is no difference in contributions to the public good between endogenously and exogenously implemented contribution schemes. The positive effect of the choice of institutions is, nevertheless, in line with the existing literature postulating (weak) evidence for enhancing cooperation through voting.

With respect to the remaining hypotheses derived from standard preferences subjects' votes in the collective-choice phase of the Multi-Phase Game are largely predictable by self-interest and, therefore, we cannot reject hypothesis H2. Low- and middle-endowed players vote more frequently for the eqpay and propcont than for the eqcont scheme. On the other hand, subjects with high initial endowment vote more frequently for the eqcont scheme. This observation is consistent with the "fairness bias" between the view of an impartial spectator and a stakeholder (e.g., Johansson-Stenman and Konow 2010). Empirical studies on the role of burden sharing rules among delegates in international climate negotiations confirm tendencies of a self-interested use of fairness arguments in international climate policy (Lange et al. 2007, Lange et al. 2010, Hjerpe et al. 2011). Furthermore, we find groups in most cases to use the opportunity to agree upon a common rule-based contribution scheme in the collective- 
choice phase of the Multi-Phase Game. What cannot be explained by standard preferences is that the eqpay scheme is under majority and unanimity rule voting the most frequently chosen contribution scheme. However, this could be explained by allowing for other-regarding preferences, more precisely inequality aversion.

Regarding the average contribution levels, we cannot reject hypothesis H1. All endogenously and exogenously implemented rule-based contribution schemes (eqcont, eqpay and propcont) are effective in increasing the level of contributions to the public good. Remarkably, all rulebased contribution schemes counteract the characteristic downward trend in contributions to the public good observed in the VCM - exogenously or endogenously implemented.

What remains unanswered is that contributions in case the eqpay scheme is chosen by unanimity are higher compared to a situation in which the scheme is chosen by majority and that contributions are lower in case the VCM is implemented endogenously compared to an exogenously implemented VCM. One possible explanation could be that subjects do not want to be overruled in the collective-choice phase of the Multi-Phase Game and behave accordingly uncooperative in case they are. This would explain our findings in the endogenously implemented eqpay scheme. Here, overruled type40 players contribute less than type40 players who could implement the eqpay scheme as their favored rule-based contribution scheme. We conclude that in the by majority implemented eqpay scheme overruled type40 players decrease the average contributions to the public good. In the endogenously implemented VCM, one could argue that all group members are overruled. No one could implement his favored rule-based contribution scheme and behave accordingly uncooperative. Nevertheless, the significant low level of contributions to the public good in the endogenously implemented VCM could be driven by a sample selection effect induced by the collective-choice phase of the Multi-Phase Game. In our experimental design, we cannot exclude that the endogenously implemented VCM acts as the collection point for uncooperative participants who could not manage to agree upon a common rule-based contribution scheme.

In more general terms, our results show that it may be important how a policy or an institutional mechanism is implemented, i.e., whether it is implemented endogenously or exogenously. We find that the choice of an institution itself has an effect on cooperation: A positive effect exists in case agents are able to agree in an election upon a common mechanism whereas a negative effect occurs in case agents have to accept a mechanism they have not voted for. From this we conclude, that letting groups of heterogeneous agents choose their institutional frameworks for themselves offers both chances and risks, depending on 
whether agents receive what they voted for or have to accept the decision of others. Of course, this implication has to be treated with caution because our experimental design shows at least two limitations. Firstly, our design automatically enforces the binding minimum contribution levels. Therefore, our design does not take into account that sovereign agents may deviate from initially accepted contribution patterns. Secondly, our experiment does not allow us to control for individual selection into a specific mechanism. It would be interesting to control for possible selection effects in future work by, for example, extending our experimental setting according to the design suggested by Dal Bó et al. (2013). Furthermore, one potentially fruitful extension would be to relax the assumption that all agents are fully informed about the cost and benefits from investing into the public good, i.e., the voting procedure could take place behind a "veil of ignorance” (Rawls 1971). 


\section{References}

Balafoutas, L., M. Kocher, L. Putterman, and M. Sutter. 2013. "Equality, Equity and Incentives: An experiment.” European Economic Review, 60: 32-51.

Barrett, S. 2003. "Environment and Statecraft: The Strategy of Environmental TreatyMaking.” Oxford University Press, New York.

Blanco, M., D. Engelmann, and H. Normann. 2011. "A Within-Subject Analysis of OtherRegarding Preferences.” Games and Economic Behavior, 72: 321-338.

Chambers, R. 1980. "Basic Concepts in the Organization of Irrigation.” In Coward, Jr., E.W. (Ed.), Irrigation and Agricultural Developments in Asia: Perspective from the Social Sciences. Cornell University Press, Ithaca and London.

Dal Bó, P., A. Foster, and L. Putterman. 2010. "Experimental Evidence on the Effect of Democracy.” American Economic Review, 100: 2205-2229.

Dannenberg, A., A. Lange, and B. Sturm. 2014. "Participation and Commitment in Voluntary Coalitions to Provide Public Goods.” Economica, 81: 257-275.

Dayton-Johnson, J. 2000a. "The Determinants of Collective Action on the Local Commons: A Model with Evidence from Mexico.” Journal of Development Economics, 62: 181-208.

Dayton-Johnson, J. 2000b. "Choosing Rules to Govern the Commons: A Model with Evidence from Mexico.” Journal of Economic Behavior \& Organization, 42: 19-41.

Endres, A. 1997. "Negotiating a Climate Convention - The Role of Prices and Quantities". International Review of Law and Economics, 17: 147-156.

Endres, A. and M. Finus. 1999. "International Environmental Agreements: How the Policy Instrument Affects Equilibrium Emissions and Welfare." Journal of Institutional and Theoretical Economics, 155: 527-550.

Ertan, A., T. Page, and L. Putterman. 2009. "Who to Punish? Individual Decisions and Majority Rule in Mitigating the Free Rider Problem.” European Economic Review, 53: 495-511.

Fehr, E. and K. Schmidt. 1999. "A Theory of Fairness, Competition and Cooperation.” Quarterly Journal of Economics, 114: 817-868.

Fischbacher, U. 2007. "Z-Tree: Zurich Toolbox for Ready-Made Economic Experiments.” Experimental Economics, 10: 171-178.

Greiner, B. 2004. "The Online Recruitment System ORSEE 2.0 - A Guide for the Organization of Experiments in Economics.” Working Paper Series in Economics, No. 10, University of Cologne.

Hamman, J., R. Weber, and J. Woon. 2011. ”An Experimental Investigation of Electoral Delegation and the Provision of Public Goods.” American Journal of Political Science, 55: 738-752.

Hjerpe, M., Å. Löfgren, B. Linnér, and M. Hennlock. 2011. "Common Ground for Effort Sharing? Preferred Principles for Distributing Climate Mitigation Efforts." Working Papers in Economics No. 491, University of Gothenburg.

Johansson-Stenman, O. and J. Konow. 2010. "Fair Air: Distributive Justice and Environmental Economics.” Environmental and Resource Economics, 46, 147-166.

Kesternich, M., A. Lange, and B. Sturm. 2014a. "The Impact of Burden Sharing Rules on the Voluntary Provision of Public Goods.“ Journal of Economic Behavior \& Organisation, 105; 107-123.

Kesternich, M., A. Lange, and B. Sturm. 2014b "On the Performance of Rule-based Contribution Schemes Under Endowment Heterogeneity.” ZEW Discussion Paper No. 14055.

Kesternich, M., A. Löschel, and A. Ziegler. 2014c „Negotiating Weights for Burden Sharing Rules among Heterogeneous Parties: Empirical Evidence from a Survey among Delegates in International Climate Negotiations.“ ZEW Discussion Paper No. 14-031. 
Kosfeld, M., A. Okada, and A. Riedl. 2009. "Institution Formation in Public Goods Games.“ American Economic Review, 99: 1335-1355.

Lange, A., Löschel, A., Vogt, C., and A. Ziegler. 2010. "On the Self-Interested use of Equity in International Climate Negotiations.” European Economic Review, 54, 359-375.

Lange, A., Vogt, C., and A. Ziegler. 2007. "On the Importance of Equity in International Climate Policy: An Empirical Analysis.” Energy Economics, 29, 545-562.

Ledyard, J. 1995. "Public Goods: A Survey of Experimental Research.” In Kagel, J and A. Roth (Eds.), The Handbook of Experimental Economics, Princeton University Press: 111 194.

Margreiter, M., M. Sutter, and D. Dittrich. 2005. "Individual and Collective Choice and Voting in Common Pool Resource Problem with Heterogeneous Actors.” Environmental and Resource Economics, 32: 241- 271.

Mill, J. 1848. “On the General Principles of Taxation.” In: Ashley, W.J. (Ed.), Principles of Political Economy with some of their Applications to Social Philosophy, Longmans, Green and Co, London, UK.

Nordhaus, W. 2010. "Economic Aspects of Global Warming in a Post-Copenhagen Environment.” Proceedings of the National Academy of Sciences of the United States of America, 107: 11721 - 11726.

Orzen, H. 2008. "Fundraising Through Competition: Evidence from the Lab." CeDEx Discussion Paper, No. 2008-11, University of Nottingham.

Ostrom, E. 1990. "Governing the Commons: The Evolution of Institutions for Collective Action.” Cambridge University Press, New York.

Posner, E. and D. Weisbach. 2010. "Climate Change Justice.” Princeton University Press.

Rawls, J. 1971. “A Theory of Justice.” Harvard University Press, Cambridge MA.

Sutter, M., S. Haigner, and M. Kocher. 2010. "Choosing the Carrot or the Stick? Endogenous Institutional Choice in Social Dilemma Situations." Review of Economic Studies, 77: 1540-1566.

Tyran, J. and L. Feld. 2006. "Achieving Compliance when Legal Sanctions are NonDeterrent.” Scandinavian Journal of Economics, 108: 135-156.

Vollan, B., Y. Zhou, A. Landmann, B. Hu, and C. Herrmann-Pillath. 2013. "Cooperation under Democracy and Authoritarian Norms." Working Papers in Economics and Statistics, No. 2013-14, University of Innsbruck.

Walker, J., R. Gardner, A. Herr, and E. Ostrom. 2000. "Collective Choice in the Commons: Experimental Results on Proposed Allocation Rules and Votes.” The Economic Journal, 110: 212-234. 


\section{Appendix}

\section{Appendix A: Tables}

Table A.1: Experimental Design

\begin{tabular}{|c|c|c|c|}
\hline & Treatment & Phases & $\begin{array}{c}\text { No. of Subjects } \\
\text { (Ind. Obs.) }\end{array}$ \\
\hline \multirow{4}{*}{ 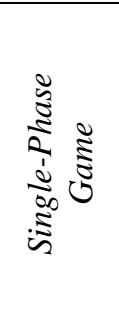 } & $V C M$ & contribution & $\begin{array}{c}45 \\
(15)\end{array}$ \\
\hline & eqpay & contribution & $\begin{array}{c}45 \\
(15)\end{array}$ \\
\hline & eqcont & contribution & $\begin{array}{c}48 \\
(46)\end{array}$ \\
\hline & propcont & contribution & $\begin{array}{c}48 \\
(12)\end{array}$ \\
\hline \multirow{2}{*}{ 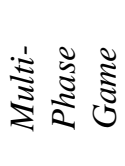 } & majority & $\begin{array}{l}\text { collective-choice } \\
\text { and contribution }\end{array}$ & $\begin{array}{c}87 \\
(29)\end{array}$ \\
\hline & unanimity & $\begin{array}{l}\text { collective-choice } \\
\text { and contribution }\end{array}$ & $\begin{array}{c}93 \\
(31)\end{array}$ \\
\hline
\end{tabular}

Notes: Players participate in groups of 3 in a repeated public goods game with overall 10 periods (excluding trial periods) and a marginal per capita return from investing into the public good of 0.6. Each group consists of one player with an initial endowment of 20, 30 , and $40 \mathrm{LD}$. In the collective-choice phase, subjects vote for the proposed rule-based contribution schemes (eqcont, eqpay and propcont). To be adopted, a rule-based contribution scheme must receive 2 out of 3 votes (majority rule) and 3 out of 3 votes (unanimity rule). If a rule-based contribution scheme is adopted it is implemented in the contribution phase. If no rule-based contribution scheme is adopted a VCM is implemented in the contribution phase.

Table A.2: Theoretical Predictions according to Standard Preferences

\begin{tabular}{lcccccrrr}
\hline & $Q^{\text {min }}$ & $q_{\text {type20 }}$ & $q_{\text {type } 30}$ & $q_{\text {type } 40}$ & $\pi_{\text {type } 20}$ & $\pi_{\text {type } 30}$ & $\pi_{\text {type } 40}$ & $\Pi$ \\
\hline VCM & 0 & 0 & 0 & 0 & 20 & 30 & 40 & 90 \\
eqcont & 78 & 20 & 29 & 29 & 46.8 & 47.8 & 57.8 & 152.4 \\
eqpay & 90 & 20 & 30 & 40 & 54 & 54 & 54 & 162 \\
propcont & 90 & 20 & 30 & 40 & 54 & 54 & 54 & 162 \\
\hline
\end{tabular}

Notes: $Q^{\min }=$ binding minimum group contribution level; $q_{i}=$ individual contribution of player $i$ to the public good; $\pi_{i}=$ individual payoff of player $i$; $\Pi$ group payoff.

Table A.3: Test Between Types (Binomial Test): Individually Chosen Rule-Bases Contribution Schemes

\begin{tabular}{lllllll}
\hline & eqcont & eqpay & \multicolumn{2}{c}{ propcont } \\
type20 & $\begin{array}{c}\text { type30 } \\
\text { type }\end{array}$ & type20 & type30 & type20 & type30 \\
\hline type30 & $>$ & & $<* * *$ & \multicolumn{4}{c}{$>* * *$} & $<* *$ \\
\hline
\end{tabular}

Notes: Votes in all three voting rounds for a rule-based contribution scheme serve as one observation. We compare rows with columns, ${ }^{*} \mathrm{p}<0.1,{ }^{* *} \mathrm{p}<0.05$ and ${ }^{* * *} \mathrm{p}<0.01$. Example: type 40 players vote less frequently for the eqpay scheme compared to type 20 players, this difference is significant at the $1 \%$-level. 
Table A.4: Implemented Contribution Schemes in the Multi-Phase Game (Binomial Test)

\begin{tabular}{llll}
\hline & VCM & eqpay & eqcont \\
\hline eqpay & $>* * *$ & & \\
eqcont & $<* * *$ & $<* * *$ & \\
propcont & $>$ & $<* * *$ & $>* * *$ \\
\hline Nores: Con
\end{tabular}

Notes: Contribution schemes chosen by a group serves as one observation. We compare rows with columns, $* \mathrm{p}<0.1, \quad * * \mathrm{p}<0.05$ and $* * * \mathrm{p}<0.01$. Example: eqpay is more frequently implemented compared to eqcont, this difference is significant at the $1 \%$-level.

Table A.5: Test Between Rounds (Binomial Test): Chosen Rule-Based Contribution Schemes

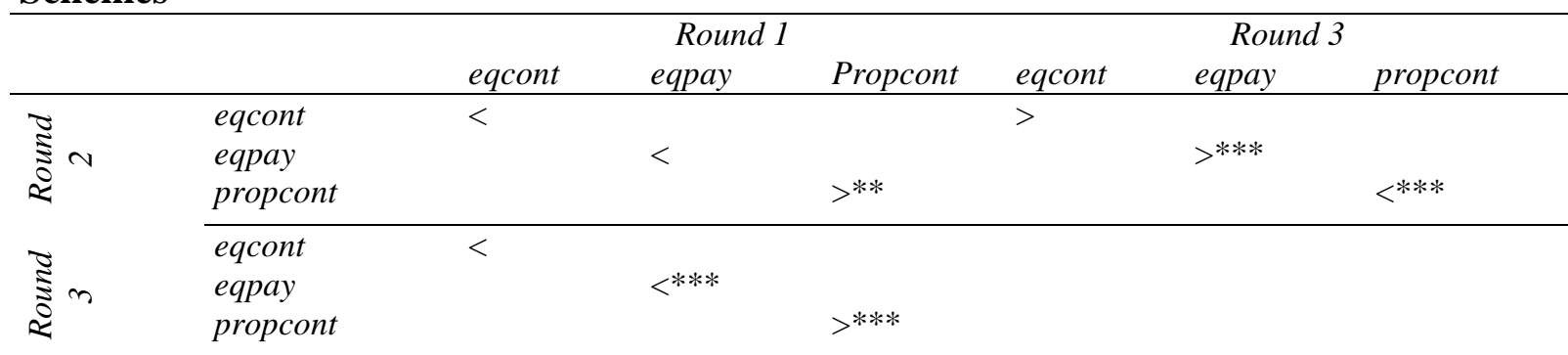

Notes: Votes in each of the three voting rounds for a rule-based contribution scheme serve as one observation. We compare rows with columns, ${ }^{*} \mathrm{p}<0.1,{ }^{* *} \mathrm{p}<0.05$ and ${ }^{* * *} \mathrm{p}<0.01$. Example: participants vote less frequently for the eqpay scheme in the third voting round compared to the first voting round, this difference is significant at the $5 \%$-level. 
Table A.6: Summary Statistic of all Contribution Schemes: Average Contributions and Payoffs per Group in Endogenously and Exogenously Implemented Contribution Schemes

\begin{tabular}{|c|c|c|c|c|c|c|c|c|}
\hline & $\begin{array}{l}\text { ex- } \\
V C M\end{array}$ & $\begin{array}{l}\text { end- } \\
\text { VCM }\end{array}$ & $\begin{array}{l}\text { ex- } \\
\text { eqcont }\end{array}$ & $\begin{array}{l}\text { end- } \\
\text { eqcont }\end{array}$ & $\begin{array}{l}\text { ex- } \\
\text { eqpay }\end{array}$ & $\begin{array}{l}\text { end- } \\
\text { eqpay }\end{array}$ & $\begin{array}{l}\text { ex- } \\
\text { propcont }\end{array}$ & $\begin{array}{l}\text { end- } \\
\text { propcont }\end{array}$ \\
\hline \multicolumn{9}{|c|}{ All Periods } \\
\hline$q_{\text {all }}$ & 17.45 & 8.14 & 21.20 & 20.33 & 21.45 & 26.32 & 24.29 & 22.48 \\
\hline$q_{\text {type } 20}$ & 13.13 & 6.74 & 16.73 & 15.74 & 13.6 & 17.07 & 16.58 & 15.27 \\
\hline$q_{\text {type } 30}$ & 17.75 & 10.20 & 21.91 & 21.35 & 21.31 & 26.04 & 24.21 & 22.49 \\
\hline$q_{\text {type } 40}$ & 21.46 & 7.49 & 24.96 & 23.89 & 29.45 & 35.84 & 32.09 & 29.68 \\
\hline$\pi_{\text {all }}$ & 43.96 & 36.51 & 46.96 & 46.26 & 47.16 & 51.05 & 49.43 & 47.99 \\
\hline$\pi_{\text {type } 20}$ & 32.27 & 27.91 & 41.44 & 40.85 & 45.02 & 50.30 & 47.15 & 45.19 \\
\hline$\pi_{\text {type } 30}$ & 43.65 & 34.46 & 46.25 & 45.24 & 47.30 & 51.33 & 49.52 & 47.97 \\
\hline$\pi_{\text {type } 40}$ & 49.95 & 47.17 & 53.20 & 52.70 & 49.17 & 51.53 & 51.63 & 50.79 \\
\hline \multicolumn{9}{|c|}{ Last Five Periods } \\
\hline$q_{\text {all }}$ & 15.58 & 6.63 & 22.14 & 20.91 & 21.61 & 27.27 & 24.43 & 23.70 \\
\hline$q_{\text {type } 20}$ & 11.64 & 5.75 & 17.30 & 15.83 & 14.16 & 17.70 & 16.74 & 15.82 \\
\hline$q_{\text {type } 30}$ & 16.06 & 8.37 & 23.33 & 22.18 & 21.32 & 26.93 & 24.38 & 23.74 \\
\hline$q_{\text {type } 40}$ & 19.04 & 5.77 & 25.81 & 24.72 & 29.36 & 37.18 & 32.19 & 31.54 \\
\hline$\pi_{\text {all }}$ & 42.47 & 35.30 & 47.72 & 46.73 & 47.29 & 51.82 & 49.55 & 48.96 \\
\hline$\pi_{\text {type20 }}$ & 36.40 & 26.19 & 42.56 & 41.81 & 44.74 & 51.39 & 47.24 & 46.84 \\
\hline$\pi_{\text {type } 30}$ & 41.99 & 33.57 & 46.53 & 45.46 & 47.58 & 52.16 & 49.60 & 48.92 \\
\hline \multicolumn{9}{|c|}{ Last Period } \\
\hline$q_{\text {all }}$ & 8.91 & 2.98 & 23.29 & 21.60 & 21.00 & 27.64 & 24.38 & 26.27 \\
\hline$q_{\text {type } 20}$ & 7.67 & 2.21 & 18.33 & 16.00 & 14.67 & 18.32 & 17.45 & 17.54 \\
\hline$q_{\text {type } 30}$ & 10.73 & 6.00 & 24.47 & 23.40 & 20.33 & 26.79 & 23.81 & 26.31 \\
\hline$q_{\text {type } 40}$ & 8.33 & 0.71 & 27.07 & 25.40 & 28.00 & 37.82 & 31.86 & 34.98 \\
\hline$\pi_{\text {all }}$ & 37.13 & 32.38 & 48.63 & 47.28 & 46.80 & 52.11 & 49.50 & 51.02 \\
\hline$\pi_{\text {type } 20}$ & 28.37 & 23.14 & 43.59 & 42.88 & 43.13 & 51.44 & 46.42 & 49.76 \\
\hline$\pi_{\text {type } 30}$ & 35.31 & 29.36 & 47.45 & 45.48 & 47.47 & 52.97 & 50.06 & 50.98 \\
\hline$\pi_{\text {type } 40}$ & 47.71 & 44.64 & 54.85 & 53.48 & 49.80 & 51.94 & 52.02 & 52.32 \\
\hline
\end{tabular}

Notes: $q_{\text {all }}=$ average contributions per group over all 10 periods (excluding trial periods), the last five periods and in the last period; $q_{i}=$ individual contribution of player $i ; \pi=$ average profits per group over all 10 periods (excluding trial periods), the last five periods and in the last period; $\pi_{i}=$ individual payoff of player $i$. Exogenously implemented contribution schemes get the prefix ex- (i.e., ex-VCM, ex-eqcont, ex-eqpay and expropcont), endogenously implemented contribution schemes get the prefix end- (i.e., end-VCM, end-eqcont, endeqpay and end-propcont). 
Table A.7: Tests Between Treatments (MW-U test): Average Contributions per Group in Endogenously and Exogenously Implemented Contribution Schemes

\begin{tabular}{|c|c|c|c|c|c|c|c|}
\hline Treatment & $\begin{array}{l}e x- \\
V C M\end{array}$ & $\begin{array}{l}\text { end- } \\
\text { VCM }\end{array}$ & $\begin{array}{l}\text { ex- } \\
\text { eqcont }\end{array}$ & $\begin{array}{l}\text { end- } \\
\text { eqcont }\end{array}$ & $\begin{array}{l}\text { ex- } \\
\text { eqpay }\end{array}$ & $\begin{array}{l}\text { end- } \\
\text { eqpay }\end{array}$ & $\begin{array}{l}\text { ex- } \\
\text { propcont }\end{array}$ \\
\hline \multicolumn{8}{|l|}{ All Periods } \\
\hline end-VCM & $<* *$ & & & & & & \\
\hline ex-eqcont & $>$ & & & & & & \\
\hline end-eqcont & & $>*$ & $<$ & & & & \\
\hline ех-еqрау & $>$ & & $>$ & & & & \\
\hline end-eqpay & & $>* * *$ & & $>$ & $>* *$ & & \\
\hline ex-propcont & $>* *$ & & $>* *$ & & $>$ & & \\
\hline end-propcont & & $>* * *$ & & $>$ & & $<$ & $<$ \\
\hline \multicolumn{8}{|l|}{ Last Five Periods } \\
\hline end-VCM & $<* *$ & & & & & & \\
\hline ex-eqcont & $>$ & & & & & & \\
\hline end-eqcont & & $>* *$ & $<$ & & & & \\
\hline ех-еqрау & $>* *$ & & $<$ & & & & \\
\hline end-eqpay & & $>* * *$ & & $>*$ & $>*$ & & \\
\hline ex-propcont & $>* * *$ & & $>* *$ & & $>$ & & \\
\hline end-propcont & & $>* * *$ & & $>$ & & $<$ & $<$ \\
\hline \multicolumn{8}{|l|}{ Last Period } \\
\hline end-VCM & $<* *$ & & & & & & \\
\hline ex-eqcont & $>* * *$ & & & & & & \\
\hline end-eqcont & & $>* *$ & $<$ & & & & \\
\hline ех-еqрау & $>* * *$ & & $<$ & & & & \\
\hline end-eqpay & & $>* * *$ & & $>* *$ & $>*$ & & \\
\hline $\begin{array}{l}\text { ex-propcont } \\
\text { end-propcont }\end{array}$ & $>* * *$ & $>* *$ & $>*$ & & $>$ & $<$ & $>$ \\
\hline $\begin{array}{l}\text { Notes: Average } \\
10 \text { periods (exclu } \\
\text { Exogenously imp } \\
\text { propcont), endog } \\
\text { eqpay and end-pr } \\
\text { VCM, this differe }\end{array}$ & $\begin{array}{l}\text { ribution } \\
\text { g trial p } \\
\text { eented } \\
\text { usly im } \\
\text { ont). E } \\
\text { is signi }\end{array}$ & $\begin{array}{l}\text { rgroup } \\
\text { is), the } \\
\text { ibution } \\
\text { ented c } \\
\text { le: ave } \\
\text { th at the }\end{array}$ & $\begin{array}{l}\text { e as one } \\
\text { ive perio } \\
\text { emes get } \\
\text { bution sc } \\
\text { over all }\end{array}$ & $\begin{array}{l}\text { ervation } \\
\text { and in th } \\
\text { prefix } \\
\text { nes get } \\
\text { riods co }\end{array}$ & $\begin{array}{l}\text { e compa } \\
\text { st perio } \\
\text { (i.e., } e x \\
\text { refix el } \\
\text { utions }\end{array}$ & $\begin{array}{l}\text { with c } \\
1,{ }^{* *} \mathrm{p} \\
\text { ex-eqco } \\
\text { end-Vc } \\
\text { opcont }\end{array}$ & $\begin{array}{l}\text { e.g., over } \\
\mathrm{d} * * * \mathrm{p}<0 .( \\
\text { qpay and } \\
\text {-eqcont, er } \\
\text { er than in }\end{array}$ \\
\hline
\end{tabular}


Table A.8: Time Trends in all Contribution Schemes: Average Contributions per Group in Endogenously and Exogenously Implemented Contribution Schemes

\begin{tabular}{|c|c|c|c|c|c|c|c|c|}
\hline Treatment & $\begin{array}{l}e x- \\
V C M\end{array}$ & $\begin{array}{l}\text { end- } \\
\text { VCM }\end{array}$ & $\begin{array}{l}\text { ex- } \\
\text { eqcont }\end{array}$ & $\begin{array}{l}\text { end- } \\
\text { eqcont }\end{array}$ & $\begin{array}{l}\text { ex- } \\
\text { eqpay }\end{array}$ & $\begin{array}{l}\text { end- } \\
\text { eqpay }\end{array}$ & $\begin{array}{l}\text { ex- } \\
\text { propcont }\end{array}$ & $\begin{array}{l}\text { end- } \\
\text { propcont }\end{array}$ \\
\hline All players & $\boldsymbol{\nabla} * * *$ & $\boldsymbol{\nabla} * * *$ & $\mathbf{\Delta}^{*}$ & & & $\Delta * * *$ & & $\mathbf{\Delta}^{*}$ \\
\hline \multicolumn{9}{|c|}{$\begin{array}{l}\text { Note: Average contributions per group in each period (excluding the trial periods) of the game serves as one } \\
\text { observation. Statistical results for time trends are based on the Jonckheere-Terpstra test. } \boldsymbol{\nabla}:=\text { decreasing } \\
\text { contributions over periods, } \boldsymbol{\Lambda}:=\text { increasing contributions over periods; }{ }^{*}<0.1, * * \mathrm{p}<0.05 \text {, } * * * \mathrm{p}<0.01 \text {. } \\
\text { Exogenously implemented contribution schemes get the prefix ex- (i.e., ex-VCM, ex-eqcont, ex-eqpay and ex- } \\
\text { propcont), endogenously implemented contribution schemes get the prefix end- (i.e., end-VCM, end-eqcont, end- } \\
\text { eqpay and end-propcont). }\end{array}$} \\
\hline
\end{tabular}

Table A.9: Summary Statistic of endogenously eqpay Scheme: Average Contributions per Group in the by Unanimity and Majority Implemented eqpay Scheme

\begin{tabular}{llll}
\hline & \multicolumn{3}{c}{ end-eqpay } \\
& all & unanimity & majority \\
\hline$q_{\text {all }}$ & 26.32 & 28.84 & 24.43 \\
$q_{\text {type20 }}$ & 17.07 & 18.87 & 15.73 \\
$q_{\text {type30 }}$ & 26.04 & 28.85 & 23.93 \\
$q_{\text {type } 40}$ & 35.84 & 38.79 & 33.62 \\
\hline Notes: $q$
\end{tabular}

over all 10 periods (excluding trial periods). 
Table A.10: Tests Between Treatments (MW-U test): Average Payoffs per Group in Endogenously and Exogenously Implemented Contribution Schemes

\begin{tabular}{|c|c|c|c|c|c|c|c|}
\hline & $\begin{array}{l}e x- \\
V C M\end{array}$ & $\begin{array}{l}\text { end- } \\
\text { VCM }\end{array}$ & $\begin{array}{l}\text { ex- } \\
\text { eqcont }\end{array}$ & $\begin{array}{l}\text { end- } \\
\text { eqcont }\end{array}$ & $\begin{array}{l}\text { ex- } \\
\text { eqpay }\end{array}$ & $\begin{array}{l}\text { end- } \\
\text { eqpay }\end{array}$ & $\begin{array}{l}\text { ex- } \\
\text { propcont }\end{array}$ \\
\hline \multicolumn{8}{|l|}{ All Periods } \\
\hline end-VCM & $<* *$ & & & & & & \\
\hline ex-eqcont & $>$ & & & & & & \\
\hline end-eqcont & & $>*$ & $<$ & & & & \\
\hline ex-еqрау & $>$ & & $>$ & & & & \\
\hline end-eqpay & & $>* * *$ & & $>$ & $>* *$ & & \\
\hline ex-propcont & $>* *$ & & $>* *$ & & $>$ & & \\
\hline end-propcont & & $>* * *$ & & $>$ & & $<$ & $<$ \\
\hline \multicolumn{8}{|l|}{ Last Five Periods } \\
\hline end-VCM & $<* *$ & & & & & & \\
\hline ex-eqcont & $>$ & & & & & & \\
\hline end-eqcont & & $>* *$ & $<$ & & & & \\
\hline ex-еqрау & $>* *$ & & $<$ & & & & \\
\hline end-eqpay & & $>* * *$ & & $>*$ & $>*$ & & \\
\hline ex-propcont & $>* * *$ & & $>* *$ & & $>$ & & \\
\hline end-propcont & & $>* * *$ & & $>$ & & $<$ & $<$ \\
\hline \multicolumn{8}{|l|}{ Last Period } \\
\hline end-VCM & $<* *$ & & & & & & \\
\hline ex-eqcont & $>* * *$ & & & & & & \\
\hline end-eqcont & & $>* *$ & $<$ & & & & \\
\hline еx-еqраy & $>* * *$ & & $<$ & & & & \\
\hline end-eqpay & & $>* * *$ & & $>* *$ & $>*$ & & \\
\hline ex-propcont & $>* * *$ & & $>*$ & & $>$ & & \\
\hline end-propcont & & $>* * *$ & & $>$ & & $<$ & $>$ \\
\hline \multicolumn{8}{|c|}{$\begin{array}{l}\text { Notes: Average payoffs per group serve as one observation. We compare rows with columns, e.g., over all } 1 \\
\text { periods (excluding trial periods), the last five periods and in the last period; }{ }^{*} \mathrm{p}<0.1,{ }^{* *} \mathrm{p}<0.05 \text { and }{ }^{* * *} \mathrm{p}<0.01 \\
\text { Exogenously implemented contribution schemes get the prefix ex- (i.e., ex-VCM, ex-eqcont, ex-eqpay and ex } \text { ex } \\
\text { propcont), endogenously implemented contribution schemes get the prefix end- (i.e., end-VCM, end-eqcont, end } \\
\text { eqpay and end-propcont). Example: averaged over all periods profits in ex-eqpay are higher than in ex-VCM, thi } \\
\text { difference is not significant at a conventional level. }\end{array}$} \\
\hline
\end{tabular}

Table A.11: Time Trends in all Contribution Schemes: Average Payoffs per Group in Endogenously and Exogenously Implemented Contribution Schemes

\begin{tabular}{|c|c|c|c|c|c|c|c|c|}
\hline Treatment & $\begin{array}{l}e x- \\
V C M\end{array}$ & $\begin{array}{l}\text { end- } \\
\text { VCM }\end{array}$ & $\begin{array}{l}\text { ex- } \\
\text { eqcont }\end{array}$ & $\begin{array}{l}\text { end- } \\
\text { eqcont }\end{array}$ & $\begin{array}{l}\text { ex- } \\
\text { eqpay }\end{array}$ & $\begin{array}{l}\text { end- } \\
\text { eqpay }\end{array}$ & $\begin{array}{l}\text { ex- } \\
\text { propcont }\end{array}$ & $\begin{array}{l}\text { end- } \\
\text { propcont }\end{array}$ \\
\hline All players & $\boldsymbol{\nabla} * * *$ & $\boldsymbol{\nabla} * * *$ & $\mathbf{\Delta}^{*}$ & & & $\underline{\Delta * * *}$ & & \\
\hline
\end{tabular}

Note: Average contributions in each period (excluding the trial periods) of the game serves as one observation. Statistical results for time trends are based on the Jonckheere-Terpstra test. $\boldsymbol{\nabla}:=$ decreasing contributions over periods, $\boldsymbol{\Delta}$ := increasing contributions over periods; $* \mathrm{p}<0.1,{ }^{*} \mathrm{p}<0.05$, *** $\mathrm{p}<0.01$. Exogenously implemented contribution schemes get the prefix ex- (i.e., ex-VCM, ex-eqcont, ex-eqpay and ex-propcont), endogenously implemented contribution schemes get the prefix end- (i.e., end-VCM, end-eqcont, end-eqpay and end-propcont). 
Table A.12: Definition of Variables

\begin{tabular}{ll}
\hline Variable & Description \\
\hline $\mathrm{q}_{\mathrm{i}}$ & Individual contribution of subject $\mathrm{i}$ to the public good \\
$\pi_{\mathrm{i}}$ & Subject i's profit \\
eqcont & $=1$ if subject i played eqcont, 0 else \\
eqpay & $=1$ if subject i played eqpay, 0 else \\
propcont & $=1$ if subject i played propcont, 0 else \\
voting & $=1$ if subject i played the Multi-Phase Game, 0 else \\
male & $=1$ if subject i is male, 0 if female \\
exp & Number of experiments subject $\mathrm{i}$ has taken part in MaXLab \\
\hline
\end{tabular}

\section{Estimation Strategy}

A series of random-effects regression models is used in order to determine individuals' contributions behavior and the corresponding payoffs. We consider individual level random effects, i.e., one observation of one individual corresponds to the panel variable and the period sets the time variable. All in all we have 3,630 observations in our econometric analysis. Due to missing sociodemographic information we have to remove three out of the 366 participants from our economic analysis. From the remaining 363 subjects we have one observation for each of the 10 payoff relevant periods. The discussion of the regression results is based on standard errors computed at individual levels.

For both, contributions to the public good and the corresponding payoffs, we use six different regression models. The first model considers only observations from Multi-Phase Game, the endogenously implemented contribution schemes (end-VCM, end-eqcont, end-eqpay and endpropcont). In contrast, the second model only considers observations form the Single-Phase Game, the exogenously implemented contribution scheme (ex-VCM, ex-eqcont, ex-eqpay and ex-propcont). The remaining four regression models consider only observations from one contribution scheme (VCM, eqcont, eqpay and propcont), endogenously or exogenously implemented. 
Table A.13: FGLS Random-Effects Regression of Contributions per Subject in Endogenously and Exogenously Implemented Contribution Schemes

\begin{tabular}{|c|c|c|c|c|c|c|}
\hline \multirow{3}{*}{$\begin{array}{l}\text { Contribution } \\
\text { Scheme }\end{array}$} & 1 & 2 & \multicolumn{2}{|r|}{4} & 5 & \\
\hline & $\begin{array}{l}1 \\
q_{i}\end{array}$ & $q_{i}$ & $\begin{array}{c}3 \\
q_{i}\end{array}$ & $\begin{array}{l}4 \\
q_{i}\end{array}$ & $\begin{array}{l}5 \\
q_{i}\end{array}$ & $q_{i}$ \\
\hline & all & all & $V C M$ & eqcont & eqpay & propcont \\
\hline Implementation & endogenously & exogenously & $\begin{array}{l}\text { endogenously } \\
\text { and } \\
\text { exogenously }\end{array}$ & $\begin{array}{l}\text { endogenously } \\
\text { and } \\
\text { exogenously }\end{array}$ & $\begin{array}{l}\text { endogenously } \\
\text { and } \\
\text { exogenously }\end{array}$ & $\begin{array}{l}\text { endogenously } \\
\text { and } \\
\text { exogenously }\end{array}$ \\
\hline \multicolumn{7}{|l|}{$\begin{array}{l}\text { Independent } \\
\text { Variables } \downarrow\end{array}$} \\
\hline eqpay & $\begin{array}{l}17.92^{* * * *} \\
(1.66)\end{array}$ & $\begin{array}{l}4.29 * \\
(2.38)\end{array}$ & & & & \\
\hline eqcont & $\begin{array}{l}11.79 * * * \\
(3.17)\end{array}$ & $\begin{array}{l}4.05^{*} \\
(2.12)\end{array}$ & & & & \\
\hline propcont & $\begin{array}{l}14.23^{* * *} \\
(2.15)\end{array}$ & $\begin{array}{l}6.92 * * * \\
(2.31)\end{array}$ & & & & \\
\hline voting & & & $\begin{array}{l}-9.38 * * * \\
(2.14)\end{array}$ & $\begin{array}{l}-1.19 \\
(2.88)\end{array}$ & $\begin{array}{l}4.63^{* *} \\
(1.98)\end{array}$ & $\begin{array}{l}-1.85 \\
(2.30)\end{array}$ \\
\hline male & $\begin{array}{l}1.44 \\
(1.54)\end{array}$ & $\begin{array}{l}2.42 \\
(1.52)\end{array}$ & $\begin{array}{l}1.16 \\
(2.20)\end{array}$ & $\begin{array}{l}4.82 * * \\
(2.15)\end{array}$ & $\begin{array}{l}0.12 \\
(1.89)\end{array}$ & $\begin{array}{l}3.63 \\
(2.32)\end{array}$ \\
\hline exp & $\begin{array}{l}-0.02 \\
(0.10)\end{array}$ & $\begin{array}{l}0.12 \\
(0.10)\end{array}$ & $\begin{array}{l}0.12 \\
(0.12)\end{array}$ & $\begin{array}{l}-0.28 * * \\
(0.13)\end{array}$ & $\begin{array}{l}0.08 \\
(0.13)\end{array}$ & $\begin{array}{l}0.14 \\
(0.18)\end{array}$ \\
\hline Constant & $\begin{array}{l}7.67 * * * \\
(1.64)\end{array}$ & $\begin{array}{l}14.94^{* * * *} \\
(2.28)\end{array}$ & $\begin{array}{l}15.58^{* * * *} \\
(2.56)\end{array}$ & $\begin{array}{l}21.51^{* * * *} \\
(1.93)\end{array}$ & $\begin{array}{l}20.72 * * * \\
(2.13)\end{array}$ & $\begin{array}{l}21.02 * * * \\
(2.18)\end{array}$ \\
\hline Observations & 1.800 & 1.830 & 870 & 600 & 1.290 & 870 \\
\hline $\begin{array}{l}\text { Number of } \\
\text { Individuals }\end{array}$ & 180 & 183 & 87 & 60 & 129 & 87 \\
\hline
\end{tabular}

Notes: $q_{i}=$ Individual contribution of subject $\mathrm{i}$ to the public good in each period (excluding the trial periods). Standard errors in parentheses, ${ }^{*} \mathrm{p}<0.1,{ }^{* *} \mathrm{p}<0.05$ and ${ }^{* * *} \mathrm{p}<0.01$. Model 1 considers observations from all endogenously implemented contribution schemes (end-VCM, end-eqcont, end-eqpay, and end-propcont). Model 2 considers all observations from exogenously implemented contribution schemes (ex-VCM, ex-eqcont, ex-eqpay, and ex-propcont). Model 3 considers observations from the endogenously and exogenously implemented VCM (end$V C M$ and $e x-V C M)$. Model 4 considers observations from the endogenously and exogenously implemented eqcont (end-eqcont and ex-eqcont). Model 5 considers observations from the endogenously and exogenously implemented eqpay (end-eqpay and ex-eqpay). Model 6 considers observations from the endogenously and exogenously implemented propcont (end-propcont and ex-propcont). 
Table A.14: FGLS Random-Effects Regression of Payoffs per Subject in Endogenously and Exogenously Implemented Contribution Schemes

\begin{tabular}{|c|c|c|c|c|c|c|}
\hline & 1 & $?$ & $?$ & & 5 & \\
\hline & $\begin{array}{c}1 \\
\pi_{i}\end{array}$ & $\pi_{i}$ & $\pi_{i}$ & $\pi_{i}$ & $\pi_{i}$ & $\pi_{i}$ \\
\hline $\begin{array}{l}\text { Contribution } \\
\text { Scheme }\end{array}$ & all & all & $V C M$ & eqcont & eqpay & propcont \\
\hline Implementation & endogenously & exogenously & $\begin{array}{c}\text { endogenously } \\
\text { and } \\
\text { exogenously }\end{array}$ & $\begin{array}{l}\text { endogenously } \\
\text { and } \\
\text { exogenously }\end{array}$ & $\begin{array}{c}\text { endogenously } \\
\text { and } \\
\text { exogenously }\end{array}$ & $\begin{array}{l}\text { endogenously } \\
\text { and } \\
\text { exogenously }\end{array}$ \\
\hline \multicolumn{7}{|l|}{$\begin{array}{l}\text { Independent } \\
\text { Variables } \downarrow\end{array}$} \\
\hline eqpay & $\begin{array}{l}14.11^{* * *} \\
(1.72)\end{array}$ & $\begin{array}{l}3.51 * \\
(1.90)\end{array}$ & & & & \\
\hline eqcont & $\begin{array}{l}9.16^{* * *} \\
(3.03)\end{array}$ & $\begin{array}{l}3.28 * \\
(1.93)\end{array}$ & & & & \\
\hline propcont & $\begin{array}{l}11.27 * * * \\
(2.07)\end{array}$ & $\begin{array}{l}5.53 * * * \\
(1.88)\end{array}$ & & & & \\
\hline voting & & & $\begin{array}{l}-7.33^{* * *} \\
(2.27)\end{array}$ & $\begin{array}{l}-1.71 \\
(2.76)\end{array}$ & $\begin{array}{l}3.44 * * * \\
(1.17)\end{array}$ & $\begin{array}{l}-1.48 \\
(1.63)\end{array}$ \\
\hline male & $\begin{array}{l}2.24 * \\
(1.28)\end{array}$ & $\begin{array}{l}1.91 \\
(1.21)\end{array}$ & $\begin{array}{l}0.39 \\
(2.30)\end{array}$ & $\begin{array}{l}3.99 * \\
(2.22)\end{array}$ & $\begin{array}{l}1.66 \\
(1.08)\end{array}$ & $\begin{array}{l}2.74 \\
(1.67)\end{array}$ \\
\hline $\exp$ & $\begin{array}{l}-0.04 \\
(0.09)\end{array}$ & $\begin{array}{l}0.13 * \\
(0.07)\end{array}$ & $\begin{array}{l}-0.06 \\
(0.13)\end{array}$ & $\begin{array}{l}-0.01 \\
(0.12)\end{array}$ & $\begin{array}{l}0.11 * \\
(0.07)\end{array}$ & $\begin{array}{l}0.10 \\
(0.13)\end{array}$ \\
\hline Constant & $\begin{array}{l}36.02 * * * \\
(1.85)\end{array}$ & $\begin{array}{l}41.64^{* * *} \\
(1.88)\end{array}$ & $\begin{array}{l}44.38 * * * \\
(2.35)\end{array}$ & $\begin{array}{l}45.12 * * * \\
(2.02)\end{array}$ & $\begin{array}{l}45.38 * * * \\
(1.44)\end{array}$ & $\begin{array}{l}47.04^{* * *} \\
(1.81)\end{array}$ \\
\hline Observations & 1.800 & 1.830 & 870 & 600 & 1290 & 870 \\
\hline $\begin{array}{l}\text { Number of } \\
\text { Groups }\end{array}$ & 180 & 183 & 87 & 60 & 129 & 87 \\
\hline
\end{tabular}

Notes: $q_{i}=$ Individual profit of subject $\mathrm{i}$ in each period (excluding the trial periods). Standard errors in parentheses, ${ }^{*} \mathrm{p}<0.1,{ }^{* *} \mathrm{p}<0.05$ and ${ }^{* * *} \mathrm{p}<0.01$. Model 1 considers observations from all endogenously implemented contribution schemes (end-VCM, end-eqcont, end-eqpay, and end-propcont). Model 2 considers all observations from exogenously implemented contribution schemes (ex-VCM, ex-eqcont, ex-eqpay, and expropcont). Model 3 considers observations from the endogenously and exogenously implemented VCM (end$V C M$ and $e x-V C M)$. Model 4 considers observations from the endogenously and exogenously implemented eqcont (end-eqcont and ex-eqcont). Model 5 considers observations from the endogenously and exogenously implemented eqpay (end-eqpay and ex-eqpay). Model 6 considers observations from the endogenously and exogenously implemented propcont (end-propcont and ex-propcont). 
Table A.15: Summary Statistic of all Contribution Schemes: Average Binding Group Minimum Contribution Level in Endogenously and Exogenously Implemented RuleBases Contribution Schemes

\begin{tabular}{|c|c|c|c|c|c|c|}
\hline & $\begin{array}{l}\text { ex- } \\
\text { eqcont }\end{array}$ & $\begin{array}{l}\text { end- } \\
\text { eqcont }\end{array}$ & $\begin{array}{l}\text { ex- } \\
\text { eqpay }\end{array}$ & $\begin{array}{l}\text { end- } \\
\text { eqpay }\end{array}$ & $\begin{array}{l}\text { ex- } \\
\text { propcont }\end{array}$ & $\begin{array}{l}\text { end- } \\
\text { propcont }\end{array}$ \\
\hline \multicolumn{7}{|c|}{ All Periods } \\
\hline$Q^{\min }$ & 61.14 & 60.66 & 61.44 & 76.55 & 69.51 & 66.67 \\
\hline$q_{\text {type } 20}^{\min }$ & 16.57 & 15.46 & 12.03 & 16.04 & 15.45 & 14.82 \\
\hline$q_{t v p e 30}^{\min }$ & 21.25 & 21.31 & 20.31 & 25.39 & 23.17 & 22.22 \\
\hline$q_{\text {type } 40}^{\min }$ & 23.32 & 23.89 & 29.10 & 35.12 & 30.89 & 29.63 \\
\hline \multicolumn{7}{|c|}{ Last Five Periods } \\
\hline$Q^{\min }$ & 64.5 & 62.20 & 63.30 & 79.98 & 70.50 & 70.85 \\
\hline$q_{\text {type } 20}^{\min }$ & 17.24 & 15.37 & 13.12 & 17.06 & 15.67 & 15.74 \\
\hline$q_{\text {type } 30}^{\min }$ & 22.57 & 22.12 & 20.89 & 26.55 & 23.50 & 23.62 \\
\hline$q_{\text {type } 40}^{\min }$ & 24.68 & 24.71 & 29.29 & 36.38 & 31.33 & 33.49 \\
\hline \multicolumn{7}{|c|}{ Last Period } \\
\hline$Q^{\min }$ & 68.80 & 64.80 & 61.20 & 80.57 & 69.94 & 78.69 \\
\hline$q_{\text {type } 20}^{\min }$ & 18.33 & 16.00 & 13.00 & 17.43 & 15.54 & 17.48 \\
\hline$q_{\text {type } 30}^{\min }$ & 24.47 & 23.40 & 20.33 & 26.75 & 23.31 & 26.23 \\
\hline$q_{t y p e 40}^{\min }$ & 26.00 & 25.40 & 27.87 & 36.39 & 31.08 & 34.98 \\
\hline
\end{tabular}

Notes: $Q^{\min }=$ binding minimum group contribution level. $q_{i}^{\min }=$ binding individual contribution level. In all 10 periods (excluding trial periods), the last five periods and in the last period. Exogenously implemented contribution schemes get the prefix ex- (i.e., ex-VCM, ex-eqcont, ex-eqpay and ex-propcont), endogenously implemented contribution schemes get the prefix end- (i.e., end-VCM, end-eqcont, end-eqpay and end-propcont).

Table A.16: Tests Between Treatments (MW-U test): Average Minimum Group Contribution Levels

\begin{tabular}{|c|c|c|c|c|c|}
\hline & $\begin{array}{l}\text { ex- } \\
\text { eqcont }\end{array}$ & $\begin{array}{l}\text { end- } \\
\text { eqcont }\end{array}$ & $\begin{array}{l}\text { ex- } \\
\text { eqpay }\end{array}$ & $\begin{array}{l}\text { end- } \\
\text { eqpay }\end{array}$ & $\begin{array}{l}\text { ex- } \\
\text { propcont }\end{array}$ \\
\hline \multicolumn{6}{|l|}{ All Periods } \\
\hline end-eqcont & $<$ & & & & \\
\hline ех-еqрау & $>$ & & & & \\
\hline end-eqpay & & $>* *$ & $>* * *$ & & \\
\hline ex-propcont & $>* * *$ & & $>*$ & & \\
\hline end-propcont & & $>$ & & $<$ & $<$ \\
\hline \multicolumn{6}{|l|}{ Last Five Periods } \\
\hline end-eqcont & $<$ & & & & \\
\hline ex-eqpay & $<$ & & & & \\
\hline end-eqpay & & $>*$ & $>$ & & \\
\hline ex-propcont & $>*$ & & $>$ & & \\
\hline end-propcont & & $>$ & & $<$ & $>$ \\
\hline \multicolumn{6}{|l|}{ Last Period } \\
\hline end-eqcont & $<$ & & & & \\
\hline ex-еqрау & $<$ & & & & \\
\hline end-eqpay & & $>* *$ & $>$ & & \\
\hline ex-propcont & $>$ & & $>$ & & \\
\hline end-propcont & & $>$ & & $<$ & $>$ \\
\hline \multicolumn{6}{|c|}{ 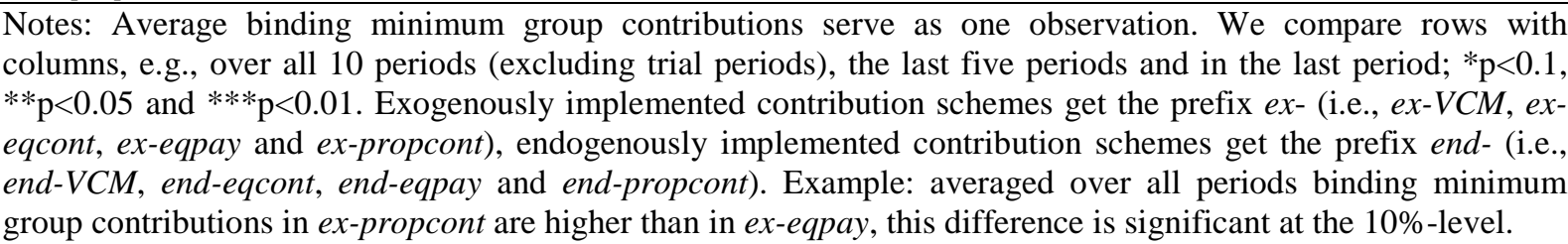 } \\
\hline
\end{tabular}




\section{Appendix B: Figures}

\section{Figure B.1: Theoretical Predictions according to Standard Preferences}
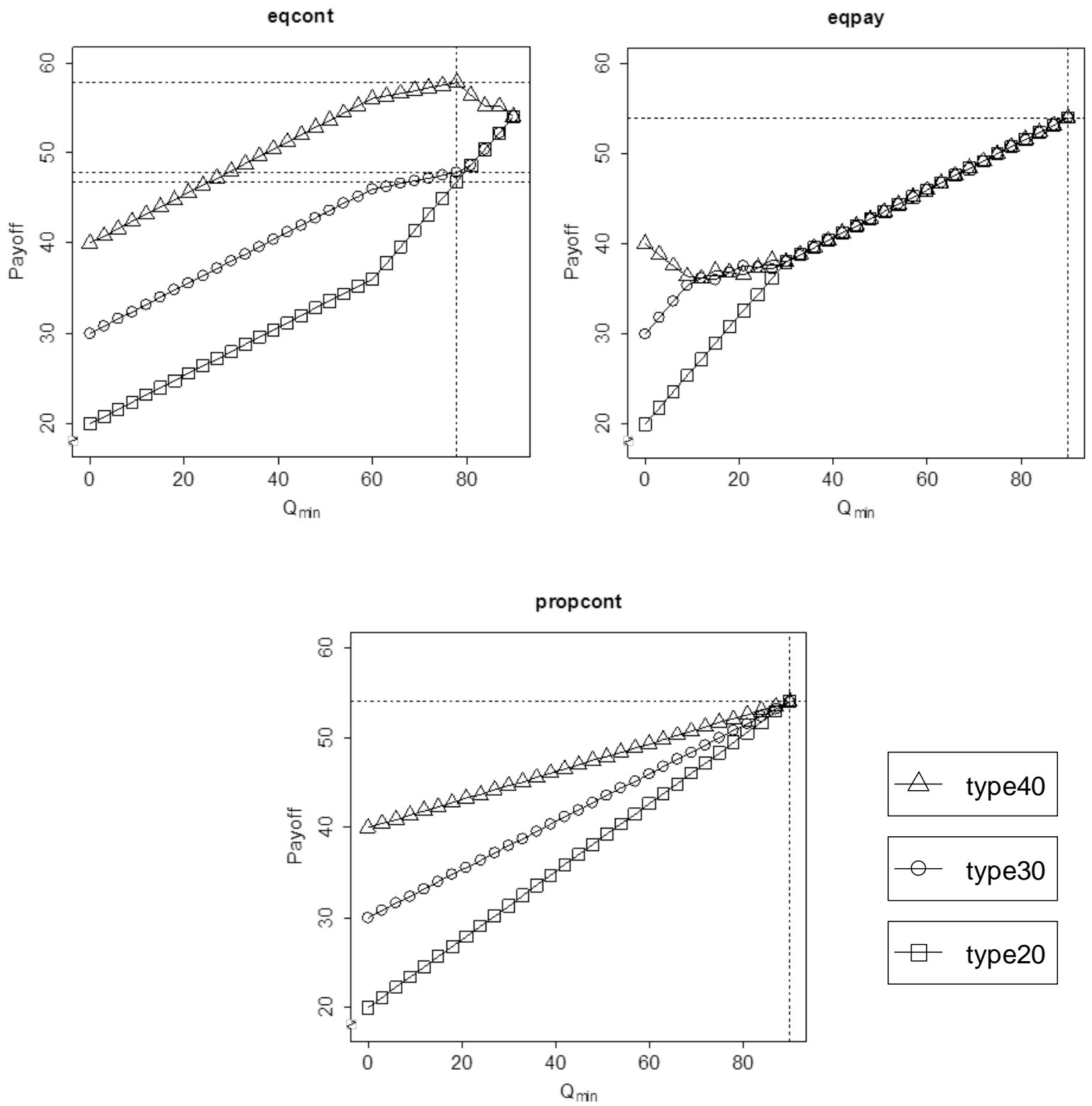

Notes: Binding minimum group contribution levels on the x-axis and corresponding payoffs for each player per group on the y-axis. Dotted lines indicate type-specific equilibrium quantities and corresponding payoffs. 
Figure B.2: Average Contributions per Group in Endogenously Implemented eqpay Schemes

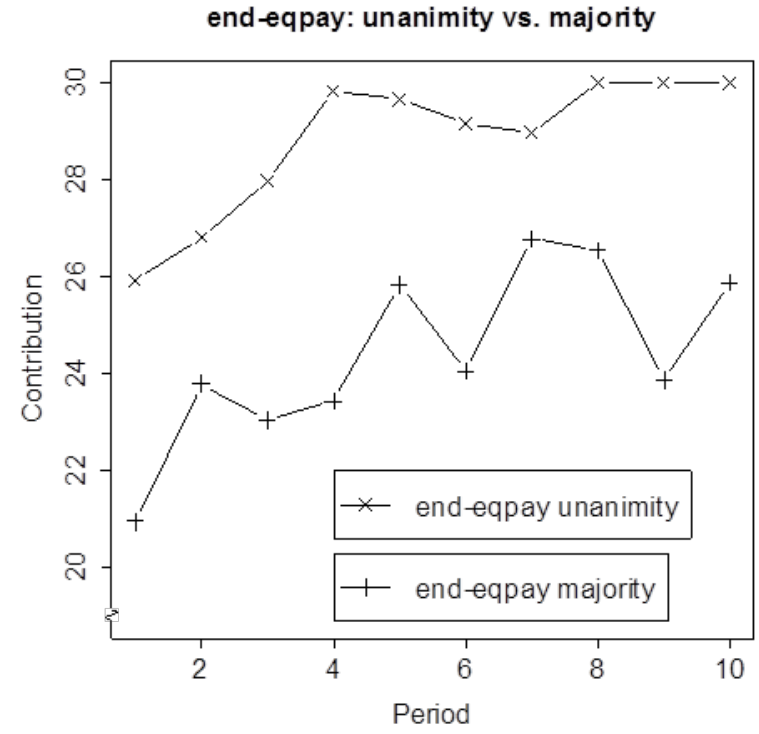

Notes: Average group contributions in each period (excluding the trial periods) of the endogenously eqpay scheme serves as one observation.

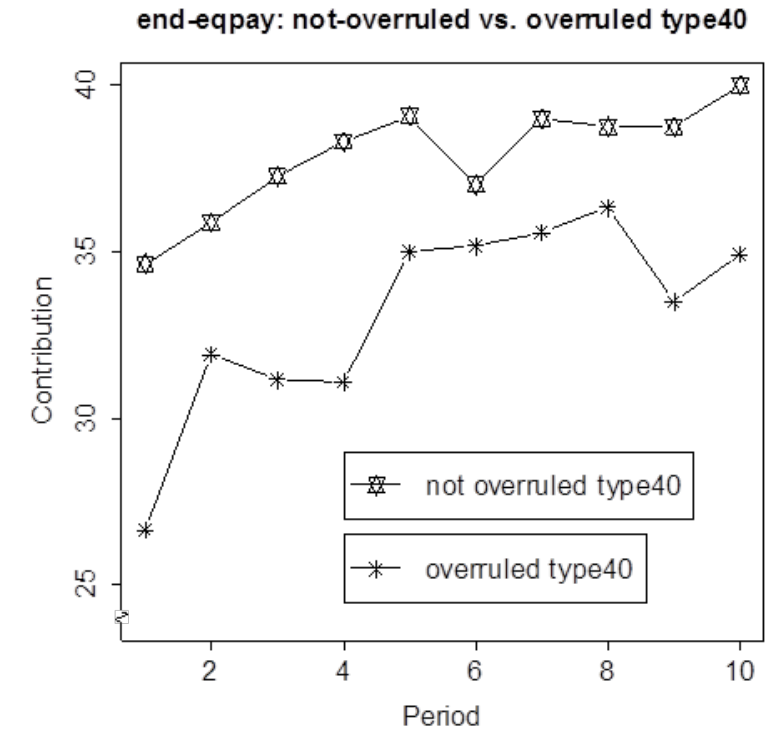

Notes: Mean contributions of overruled and not overruled type 40 players. Average contributions in each period (excluding the trial periods) of the by majority implemented eqpay scheme serves as one observation.

Figure B.3: Average Contributions per Group in the eqpay Scheme Implemented by Voting in $1^{\text {st }}$ Round or Exogenously

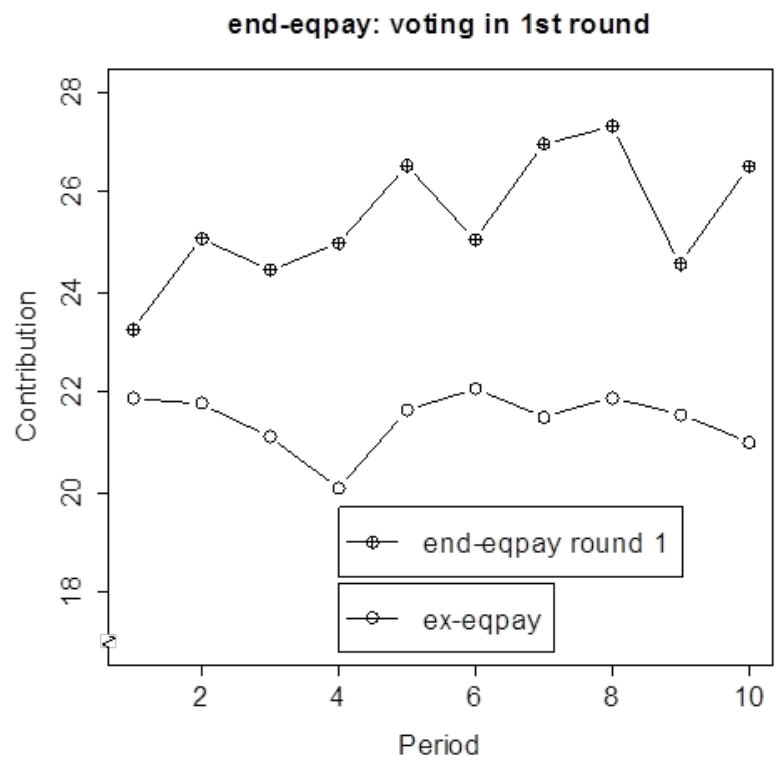

Notes: Average group contributions in each period (excluding the trial periods) of the eqpay scheme serves as one observation. 


\section{Appendix C: Theoretical Predictions}

\section{Analytical Solution according to Standard Preferences}

\section{Equal-Contribution Scheme (eqcont)}

In the eqcont scheme a subgame perfect equilibrium in weakly dominant strategies is characterized by $Q^{\min }=78, q_{\text {type } 20}^{\min }=e_{\text {type } 20}$ and $q_{\text {type } 30}^{\min }=q_{\text {type } 40}^{\min }=\frac{1}{n-1}\left(78-e_{\text {type } 20}\right)$. type20 and type30 players have a weakly dominant strategy to propose $Q^{\min }=E$ because their marginal payoff from increasing the groups' binding minimum contribution level is positive for all $Q^{\min } \in[0, E]$. In case their proposal is binding, increasing the minimum contribution until $Q^{\text {min }}=E$ would increase their payoff. In case their proposal is not binding, proposing $Q^{\min }=E$ would not hurt them. Following this logic type40 players have a weakly dominant strategy to propose $Q^{\min }=78$, because their payoff is maximized for $Q^{\min }=78$. Since the smallest proposal is binding the subgame perfect equilibrium in weakly dominant strategies given by $Q^{\min }=78$.

Since it is obligatory that $Q^{\min }$ is provided and $q_{i} \in\left[0, e_{i}\right]$ payoff functions are discontinuous and separated in three parts:

Part 1: If $Q^{\min } \in[0,60]$, players' minimum individual contribution levels are given by $q_{i}^{\min }=\frac{1}{n} Q^{\min }$ and individual payoff is given by

$$
\pi_{i}=e_{i}-\frac{1}{n} Q^{\min }+b Q^{\min }
$$

Therefore, players' marginal payoff form increasing the groups' binding minimum contribution level is given by $\frac{\partial \pi_{i}}{\partial Q^{\min }}=-\frac{1}{n}+b=-\frac{1}{3}+\frac{3}{5}=\frac{4}{15}>0$ and the marginal benefits exceeding its costs.

Part 2: If $\left.\left.Q^{\min } \in\right] 60,80\right], q_{\text {type20 }}^{\min }=e_{\text {type20 }}$ and $q_{\text {type } 30}^{\min }=q_{\text {type } 40}^{\min }=\frac{1}{n-1}\left(Q^{\min }-e_{\text {type } 20}\right)$. Therefore, type20 players' marginal payoff is given by $\frac{\partial \pi_{t y p e 20}}{\partial Q^{\min }}=b=\frac{3}{5}>0$ and for type30 as well as type 40 given by $\frac{\partial \pi_{\text {type } 30}}{\partial Q^{\min }}=\frac{\partial \pi_{\text {type } 40}}{\partial Q^{\min }}=-\frac{1}{n-1}+b=-\frac{1}{2}+\frac{3}{5}=\frac{1}{10}>0$.

Part 3: If $\left.\left.Q^{\min } \in\right] 80,90\right], q_{\text {type } 20}^{\min }=e_{\text {type } 20}, q_{\text {type } 30}^{\min }=e_{\text {type } 30}$ and $q_{\text {type } 40}^{\min }=\frac{1}{n-2}\left(Q^{\min }-\right.$ $\left.e_{\text {type } 20}-e_{\text {type } 30}\right)$. Therefore type20 and type30 players' marginal payoff is given by 
$\frac{\partial \pi_{\text {type } 20}}{\partial Q^{\min }}=\frac{\partial \pi_{\text {type } 30}}{\partial Q^{\min }}=b=\frac{3}{5}>0$ and for type40 players' given by $\frac{\partial \pi_{\text {type } 40}}{\partial Q^{\min }}=-\frac{1}{n-2}+b=$ $-1+\frac{6}{10}=-\frac{2}{5}<0$

\section{Equal-Payoff Scheme (eqpay)}

In the eqpay scheme, we expect all players to suggest full contribution levels, i.e., $Q_{i}^{\text {min }}=E$. type20 and type30 players have a weakly dominant strategy to propose $Q^{\min }=E$ and by anticipating that also type40 players have an incentive to suggest $Q^{\text {min }}=E$. Therefore we expect the groups' binding minimum contribution level to be $Q^{\min }=E$, which implies $q_{\text {type } 20}^{\min }=e_{\text {type } 20}, q_{\text {type } 30}^{\min }=e_{\text {type30 }}$ and $q_{\text {type } 40}^{\min }=e_{\text {type } 40}$.

Since we do not allow for direct redistribution of initial endowment and $q_{i} \in\left[0, e_{i}\right]$ payoff functions are discontinuous and separated in three parts.

Part 1: If $Q^{\min } \in[30,90]$ individual minimum contributions are given by $q_{i}^{\min }=e_{i}-$ $\frac{1}{n}\left(E-Q^{\text {min }}\right)$ and individual payoffs are

$$
\pi_{i}=e_{i}-\left(e_{i}-\frac{1}{n}\left(E-Q^{\min }\right)\right)+b Q^{\min }
$$

Therefore, the marginal benefits form increasing the groups' minimum contribution level exceeds its costs for all players and the marginal payoff is given by $\frac{\partial \pi_{i}}{\partial Q^{\min }}=-\frac{1}{n}+b=$ $-\frac{1}{3}+\frac{3}{5}=\frac{4}{15}>0$

Part 2: If $\left.Q^{\min } \in\right] 9,30\left[\right.$ individual minimum contributions are $q_{\text {type } 20}^{\min }=0$, $q_{\text {type } 30}^{\min }=$ $q_{\text {type } 40}^{\min }=e_{j}-\frac{1}{n-1}\left(e_{\text {type } 40}+e_{\text {type } 30}-Q^{\text {min }}\right)$. Therefore, the marginal payoff for type20 players is given by $\frac{\partial \pi_{\text {typezo }}}{\partial Q^{\min }}=b=\frac{6}{10}>0$, and for type30 as well type40 it is given by $\frac{\partial \pi_{\text {type } 30}}{\partial Q^{\min }}=\frac{\partial \pi_{\text {type } 40}}{\partial Q^{\min }}=-\frac{1}{n-1}+b=-\frac{1}{2}+\frac{3}{5}=\frac{1}{10}>0$.

Part 3: If $Q^{\min } \in[0,9]$ individual minimum contributions are $q_{\text {type } 20}^{\min }=q_{\text {type } 30}^{\min }=0$ and $q_{\text {type } 40}^{\min }=Q^{\min }$. Therefore, marginal payoffs for type20 and type30 players are given by $\frac{\partial \pi_{\text {type } 20}}{\partial Q^{\min }}=\frac{\partial \pi_{\text {type } 30}}{\partial Q^{\min }}=b=\frac{3}{5}>0$ and $\frac{\partial \pi_{\text {type40 }}}{\partial Q^{\min }}=-1+b=-1+\frac{3}{5}=-\frac{2}{5}<0$ for type40 players. 


\section{Proportional-Contribution Scheme (propcont)}

In the propcont scheme a subgame perfect equilibrium in weakly dominant strategies is characterized by $Q^{\min }=E=90$, and $q_{\text {type } 20}^{\min }=e_{\text {type } 20}, q_{\text {type } 30}^{\min }=e_{\text {type } 30}$ and $q_{\text {type } 40}^{\min }=$ $e_{\text {type } 40}$.

For all players, the benefits from increasing the binding group minimum contribution level exceeds its costs for all $Q^{\text {min }} \in[0, E]$ and it is the weakly dominant strategy for all players to propose $Q^{\min }=E$.

For all $Q^{\min } \in[0, E]$ the binding individual contribution level is given by $q_{i}^{\min }=\frac{e_{i}}{E} Q^{\min }$ and individual payoffs are given by

$$
\pi_{i}=e_{i}-\frac{e_{i}}{E} Q^{\min }+b Q^{\min }
$$

Therefore for all players the marginal benefits from increasing the groups' binding minimum contribution level exceeds its costs and the marginal payoffs are given by $\frac{\partial \pi_{i}}{\partial Q^{\min }}=-\frac{e_{i}}{E}+b=$ $-\frac{2}{9}+\frac{3}{5}=\frac{17}{45}>0$ for type20 players, $-\frac{1}{3}+\frac{3}{5}=\frac{4}{15}>0$ for type30 players and $-\frac{4}{9}+\frac{3}{5}=\frac{7}{45}>$ 0 for type 40 players. 


\section{Appendix D: Voting Behaviour and Other Regarding Preferences}

F\&S introduce the idea that actors may be averse to inequality. They formalise the idea of inequality aversion by introducing the following utility for player $i$, given the payoffs for all other players $j$ :

$$
U_{i}\left(\pi_{i}, \pi_{j}\right)=\pi_{i}-\frac{\alpha_{i}}{N-1} \sum_{j \neq i}^{N} \max \left\{\pi_{j}-\pi_{i}, 0\right\}-\frac{\beta_{i}}{N-1} \sum_{j \neq i}^{N} \max \left\{\pi_{i}-\pi_{j}, 0\right\}
$$

For $\alpha_{i}, \beta_{i}>0$, this implies that player $i$ derives disutility from inequality. The second term in the utility function represents disutility from disadvantageous inequality (in case of $\pi_{j}>\pi_{i}$, weighted by $\alpha_{i}$ ), while the third term reflects disutility from advantageous inequality (in case of $\pi_{i}>\pi_{j}$, weighted by $\beta_{i}$ ). F\&S assume $\beta_{i}<1$ and $\alpha_{i} \geq \beta_{i}$.

By assuming that participants prefer the burden sharing rule which leads at least at one minimum group contribution level $Q^{\min } \in[0,90]$ to a strict higher expected payoff in comparison to the other rules, the preference order for the different types of players with standard preferences is as follows:

$$
\begin{aligned}
\text { type 20: } & \pi_{i, \text { eqpay }}>\pi_{i, \text { propcont }}>\pi_{i, \text { eqcont }} \\
\text { type30: } & \pi_{i, \text { eqpay }}>\pi_{i, \text { propcont }}>\pi_{i, \text { eqcont }} \\
\text { type40: } & \pi_{i, \text { eqcont }}>\pi_{i, \text { propcont }}>\pi_{i, \text { eqpay }} .
\end{aligned}
$$

Assuming standard preferences, type20 and type30 players would, thus, vote for eqpay and type40 players for eqcont. These are the predictions we derived in Section 3.2 although here we relaxed the assumption that participants take only equilibrium minimum contribution levels into account and assume that all possible minimum contribution levels $Q^{\text {min }} \in[0,90]$ are considered. Figure D.1 shows in the upper section the expected payoffs for the different types of players in the different burden sharing rules and illustrates the voting behaviour.

This pictures changes if we allow for inequality aversion and compare F\&S utility values. In particular, if we assume $\beta_{i}=0.67$, type 40 players are indifferent between eqpay and the other schemes, and both other types strictly prefer eqpay. For values $\beta_{i}>0.67$, all types of players strictly prefer eqpay. This holds independent from the value for $\alpha_{i}$. Figure D.1 shows in the lower section the utility values for the different types of players in the different burden sharing rules by assuming a F\&S utility function with $\beta_{i}=0.8$ and $\alpha_{i}=0$. 
Figure D.1: Voting Behavior According to Standard Preferences and Other-Regarding Preferences
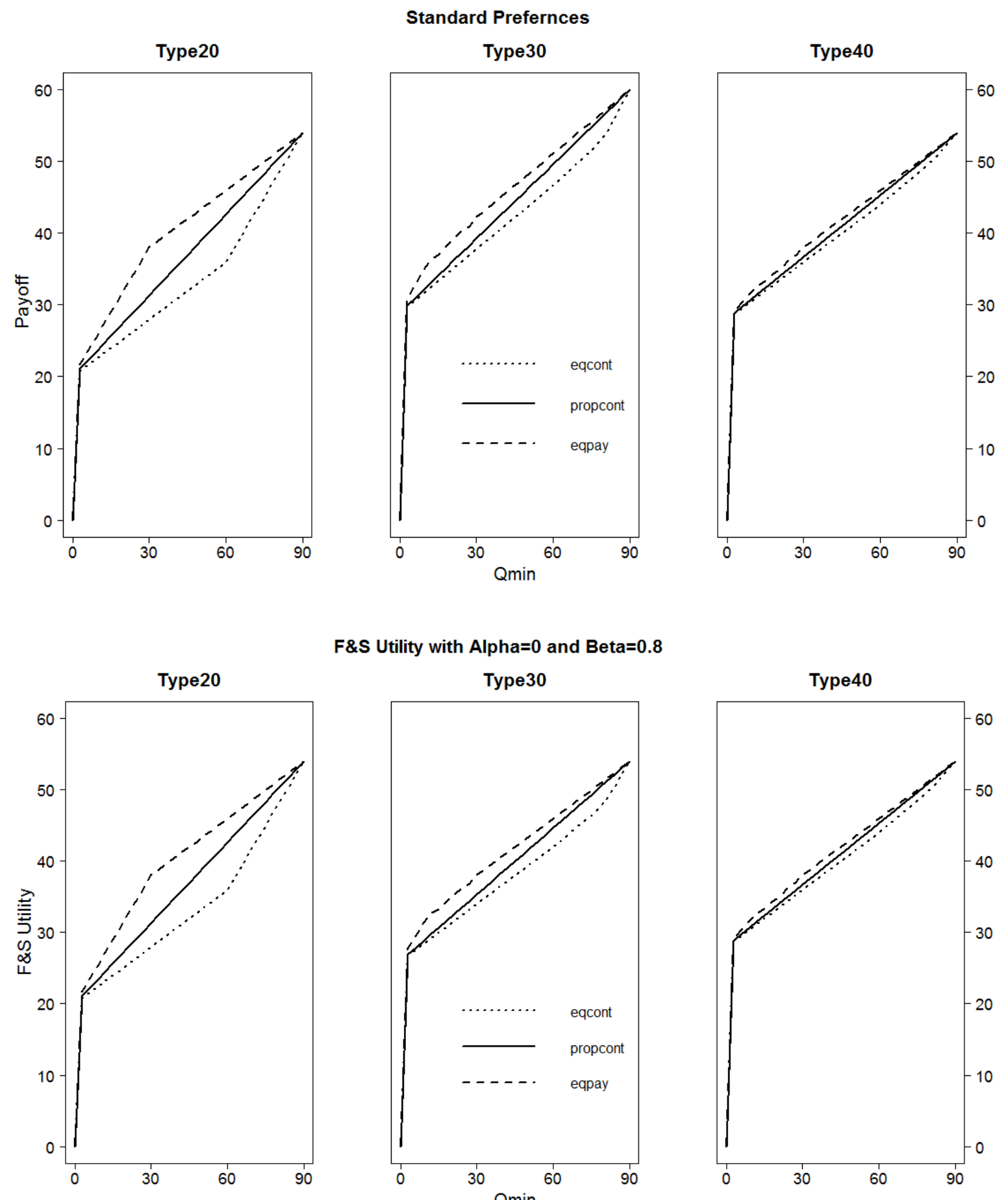

F\&S Utility with Alpha $=0$ and Beta $=0.8$
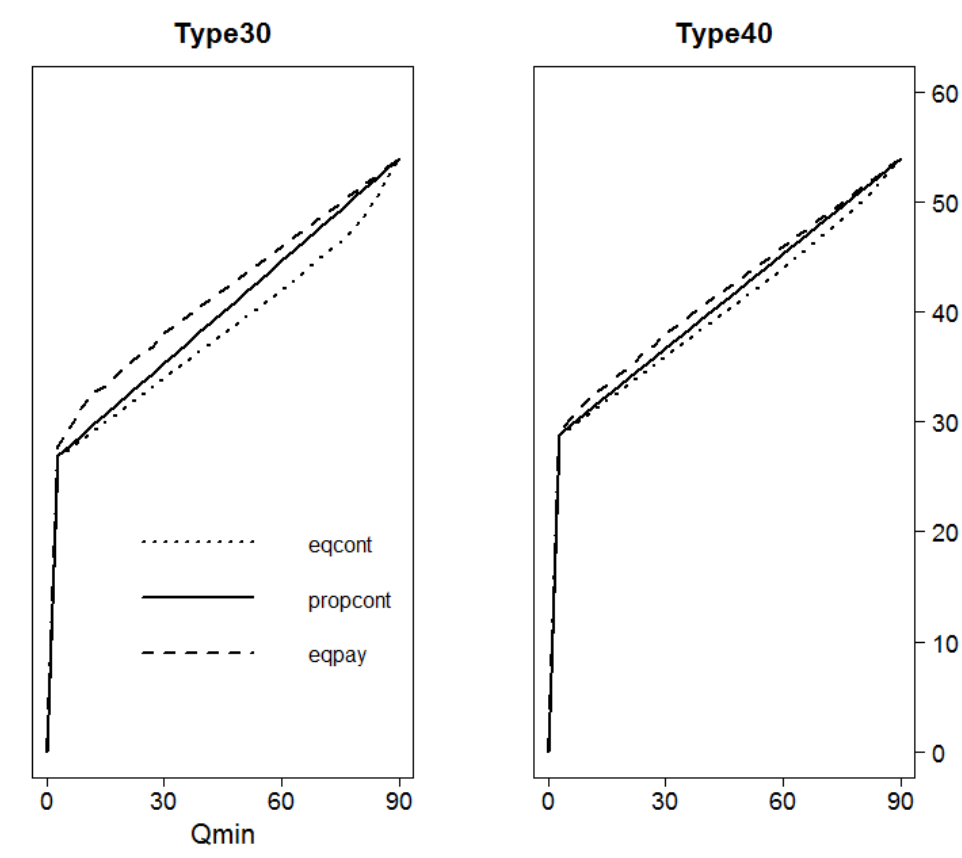


\section{Appendix E: Instructions \\ Multi-Phase Game}

Please read the instructions carefully and contact us by opening the door or giving a hand signal if you have any questions. Please do not talk to each other and do not use any electronic devices such as mobile phones, smart phones, or the like throughout the whole experiment. In the experiment you are now taking part in, you can earn money depending on your decisions and those of your teammates. Your payoff from the experiment is calculated in LaborDollars (LD) and the exchange rate between $€$ and LD is 1:3, i.e., 3 LD equals $1 €$.

During the experiment, you make your decisions anonymously. Only the experimenter will know your identity and your data will be treated confidentially. This experiment consists of two parts that will be carried out consecutively: (1) voting and (2) game. Please read the rules of the game in the following. After that, you will get details about the voting procedure.

\section{Rules of the Game}

Three players will take part in the game, i.e., apart from you, there are two other players. All in all, your group of three players has an initial endowment of $90 \mathrm{LD}$. One of the players is provided with an initial endowment of 20 LD ("type20" in the following). Another player ("type30") is provided with an initial endowment of $30 \mathrm{LD}$ and another one is provided with an initial endowment of $40 \mathrm{LD}$ ("type40"). Whether you are type20, type30, or type40 will be drawn by lot and announced before the voting.

Your task in the game (which is the same for your teammates in your group) is to decide what amount of LD you are willing to contribute to a joint project. Your contribution, q, to the project can range between 0 and $20 \mathrm{LD}$ if you are type20. Your contribution can range between 0 and $30 \mathrm{LD}$ if you are type30 and between 0 and $40 \mathrm{LD}$ if you are type40.

The individual payoff (in LD) for each one of the three players is derived as follows:

Payoff $=$ (initial endowment of player - contribution of player $)+0.6 \cdot($ total sum of contributions by all players)

Assume you are type20, then your payoff (in LD) is:

Payoff $=(\mathbf{2 0}-$ your contribution $)+0.6 \cdot($ total sum of contributions by all players $)$

That means, if, for example, the other two players contribute a total of $70 \mathrm{LD}$ and you contribute $10 \mathrm{LD}$ to the project, then your payoff is

Payoff $=(20-10)+0,6 \cdot(70+10)=58$

Whereas, if the other two players contribute a total of $70 \mathrm{LD}$ and you contribute nothing, your payoff is

Payoff $=(20-0)+0,6 \cdot(70+0)=62$

If you are type30, your payoff is (in LD):

Payoff $=(\mathbf{3 0}-$ your contribution $)+0.6 \cdot($ total sum of contributions by all players $)$

If you are type40, your payoff is (in LD):

Payoff $=(\mathbf{4 0}-$ your contribution $)+0.6 \cdot($ total sum of contributions by all players $)$

The game has two stages. In stage 1, you decide which minimum contribution, $\mathrm{Q}_{\min }$, the group shall make. The other players in your group state as well which minimum contribution, $\mathbf{Q}_{\min }$, they would like to have for the group.

The minimum of the suggestions, $\min \left(\mathbf{Q}_{\mathbf{m i n}}\right)$, is set as the minimum contribution of the group. Then in stage 2, you decide about your contribution, $q$, to the project, whereby for every player a lower limit, $q_{\text {min }}$, for the individual contribution is calculated from $\min \left(\mathrm{Q}_{\min }\right)$ according to a specific rule.

There are three rules to vote from:

Rule 1 "Equal Contribution": $q_{\min }$ is determined from $\min \left(\mathrm{Q}_{\min }\right)$ so that the minimum contributions, $\mathrm{q}_{\mathrm{min}}$, of all players are as equal as possible* so that every player contributes at least one third of the group's minimum contribution, $\min \left(\mathrm{Q}_{\min }\right)$, i.e., $\mathrm{q}_{\min }=(1 / 3) \cdot \min \left(\mathrm{Q}_{\min }\right)$.

Rule 2 "Equal Payoff": $q_{\min }$ is determined from $\min \left(\mathrm{Q}_{\min }\right)$ so that the payoffs off all players are equal or at least adjusted as far as possible*.

Rule 3 "Proportional Contribution": $\mathrm{q}_{\min }$ is determined from $\min \left(\mathrm{Q}_{\min }\right)$ so that the minimum contributions, $\mathrm{q}_{\min }$, of all players are proportional to their initial endowment. I.e., the higher the initial endowment the higher is the minimum contribution, $\mathrm{q}_{\min }$, to the joint project by the player.

\footnotetext{
* Please note that the adjustment is subject to the condition that the minimum contribution of the group to the joint project is reached.
}

Examples for the rules 1-3 with a minimum contribution of the group $\min \left(\mathrm{Q}_{\min }\right)=45^{*}$. 


\begin{tabular}{|c|c|c|c|c|c|c|}
\hline & \multicolumn{2}{|c|}{$\begin{array}{c}\text { Rule 1 } \\
\text { "Equal Contribution" }\end{array}$} & \multicolumn{2}{c|}{$\begin{array}{c}\text { Rule 2 } \\
\text { “Equal Payoff” }\end{array}$} & \multicolumn{2}{c|}{$\begin{array}{c}\text { Rule 3 } \\
\text { "Proportional } \\
\text { Contribution” }\end{array}$} \\
\hline & $\begin{array}{c}\text { Lower limit of } \\
\text { contribution }\end{array}$ & Payoff & $\begin{array}{c}\text { Lower limit of } \\
\text { contribution }\end{array}$ & Payoff & $\begin{array}{c}\text { Lower limit of } \\
\text { contribution }\end{array}$ & Payoff \\
\hline Type20 & 15 & 32 & 5 & 42 & 10 & 37 \\
\hline Type30 & 15 & 42 & 15 & 42 & 15 & 42 \\
\hline Type40 & 15 & 52 & 25 & 42 & 20 & 47 \\
\hline
\end{tabular}

* Assumption: Each player chooses their lower limit as the contribution, i.e. $\mathrm{q}=\mathrm{q}_{\min }$.

Please use the simulator to understand the examples. On your screen you will find an Excel file named "simulator". You can enter your desired minimum contribution of the group, $\min \left(Q_{\min }\right)$, in the simulator. For each rule (rule 1 "Equal Contribution", rule 2 "Equal Payoff", rule 3 "Proportional Contribution") the individual minimum contributions, $q_{\min }$, and the corresponding payoffs to each player as well as the payoff to the group are calculated. Please note that only the corresponding minimum contributions are calculated, i.e. the minimum contribution of the group, $\min \left(\mathrm{Q}_{\min }\right)$, is distributed to the players according to the different rules. Of course, you can also contribute more than the calculated minimum contribution, but only as long as your contribution does not exceed your initial endowment.

The game consists of ten separate rounds, in which you always play the same game, you remain the same type and you interact with the same two participants. In each round, you will be informed about the proposals of the minimum contribution $\left(\mathrm{Q}_{\min 1}\right.$ to $\left.\mathrm{Q}_{\min 3}\right)$, the contributions $\left(\mathrm{q}_{1}\right.$ to $\left.\mathrm{q}_{3}\right)$ and the payoffs (payoff to payoff $f_{3}$ ) of all players in your group as well as the average values (D).

At the end of the experiment you will receive the payoff of one of the ten rounds in $€(3 \mathrm{LD}=1 €)$. The round that will be disbursed is chosen randomly. Therefore, in each round, you should act as if it was relevant to disbursement. In the beginning, there will be two trial rounds which are not relevant to disbursement.

\section{Voting}

The members of a group decide for themselves which rule will be applied for the distribution of the group's minimum contribution, $\min \left(\mathrm{Q}_{\min }\right)$. The voting is a majority vote [alternative wording for unanimity rule voting: the voting is a unanimity rule] (between rule 1 "Equal Contribution", rule 2 "Equal Payoff" and rule 3 "Proportional Contribution"), i.e. if at least two of the [alternative wording for unanimity rule voting: if all of the] three group members vote for the same rule, it will be applied. There is a maximum of three voting rounds.

If there is no rule that has received at least two of the three votes after the third voting, the game will be played without stage 1, i.e. no minimum contribution is determined and each player just states their contribution to the project and the payoffs will be determined as mentioned above.

Illustration 1 shows the course of the experiment in a nutshell.

\section{Illustration 1}

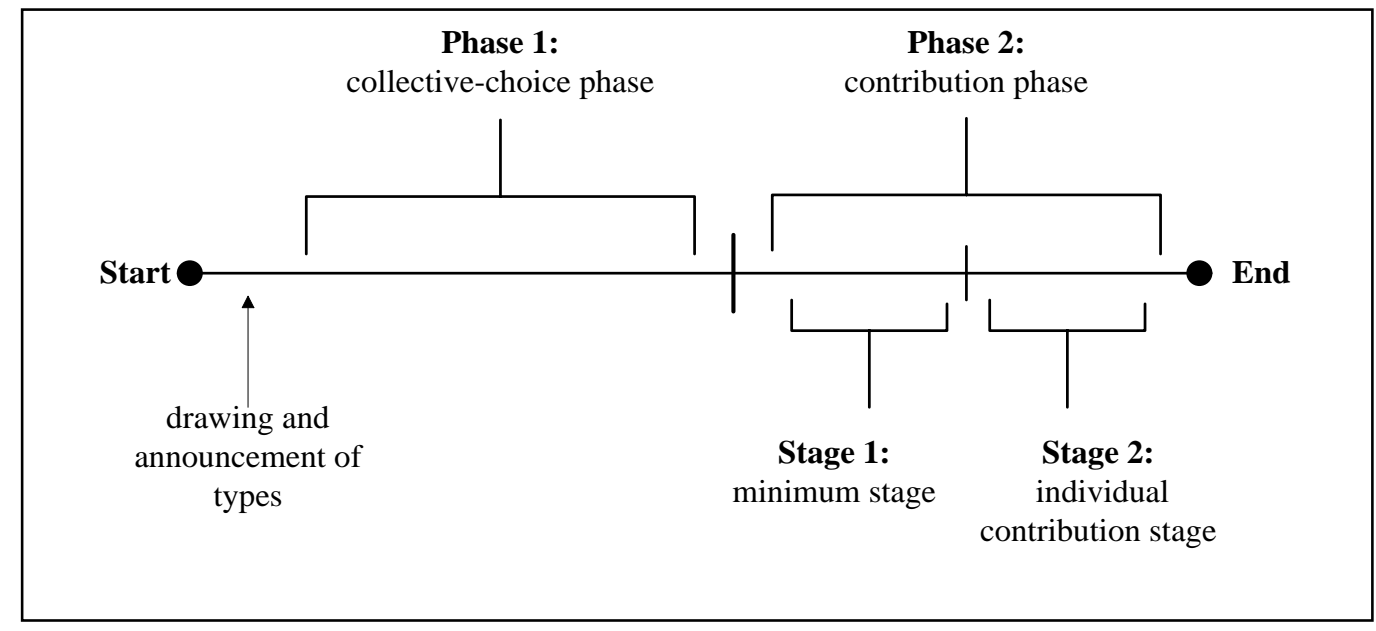




\section{Control Questions (please answer, use the simulator if necessary)}

1. Assume, the three players have stated 10,20 and 30 respectively as the proposal for the minimum contribution.

What is the group's minimum contribution $\min \left(\mathrm{Q}_{\min }\right)$ ?

The group's minimum contribution, $\min \left(\mathrm{Q}_{\min }\right)$, is:

2. Assume, the group's minimum contribution, $\min \left(\mathrm{Q}_{\min }\right)$, to the project is 30 . What is your minimum contribution and payoff if you have agreed on the following rules, if all the players contribute their minimum contribution and you are type20? (Tip: Use the simulator)

\begin{tabular}{|l|r|r|r|}
\hline Agreed rule $\rightarrow$ & $\begin{array}{c}\text { Rule 1 } \\
\text { "Equal Contribution” }\end{array}$ & $\begin{array}{c}\text { Rule 2 } \\
\text { "Equal Payoff" }\end{array}$ & $\begin{array}{c}\text { Rule 3 } \\
\text { "Proportional } \\
\text { Contribution" }\end{array}$ \\
\hline $\begin{array}{l}\text { My minimum contribution } \\
\mathrm{q}_{\text {min }}\end{array}$ & & & \\
\hline My payoff & & & \\
\hline
\end{tabular}

3. Assume, you could not agree on a rule in your group. Afterwards you make as a type30 a contribution of $20 \mathrm{LD}$. The other two players contribute $0 \mathrm{LD}$ and $10 \mathrm{LD}$. What is your payoff?

My payoff is:

4. We have the same situation as in 3.) and the other players in your group have contributed their whole initial endowment to the project. Which of the following contributions gives you the highest payoff as a type40?

(please tick)
O 0 LD
O $10 \mathrm{LD}$
O 20 LD
O $40 \mathrm{LD}$

5. We have the same situation as in 3.) and the other players in your group have contributed their whole initial endowment to the project. Which of the following contributions gives the group the highest payoff if you are a type40? (please tick)
$\mathrm{O} 0 \mathrm{LD}$
O 10 LD
O 20 LD
O $40 \mathrm{LD}$

If you have answered all the questions, please give us a sign. We will then check your answers. The game will start (with explanations on the screen) when all participants have answered the control questions correctly.

Good luck!

The MaXLab-Team 


\section{Single-Phase Game}

Please read the instructions carefully and contact us by opening the door or giving a hand signal if you have any questions. Please do not talk to each other and do not use any electronic devices such as mobile phones, smart phones, or the like throughout the whole experiment. In the experiment you are now taking part in, you can earn money depending on your decisions and those of your teammates. Your payoff from the experiment is calculated in LaborDollars (LD) and the exchange rate between $€$ and LD is 1:3, i.e., 3 LD equals $1 €$.

During the experiment, you make your decisions anonymously. Only the experimenter will know your identity and your data will be treated confidentially.

\section{Rules of the Game}

Three players will take part in the game, i.e., apart from you, there are two other players. All in all, your group of three players has an initial endowment of $90 \mathrm{LD}$. One of the players is provided with an initial endowment of 20 LD ("type20" in the following). Another player ("type30") is provided with an initial endowment of $30 \mathrm{LD}$ and another one is provided with an initial endowment of $40 \mathrm{LD}$ ("type40"). Whether you are type20, type30, or type40 will be drawn by lot and announced before the voting.

Your task in the game (which is the same for your teammates in your group) is to decide what amount of LD you are willing to contribute to a joint project. Your contribution, $\mathbf{q}$, to the project can range between 0 and $20 \mathrm{LD}$ if you are type20. Your contribution can range between 0 and $30 \mathrm{LD}$ if you are type30 and between 0 and $40 \mathrm{LD}$ if you are type 40 .

The individual payoff (in LD) for each one of the three players is derived as follows:

Payoff $=$ (initial endowment of player - contribution of player $)+0.6 \cdot($ total sum of contributions by all players)

Assume you are type20, then your payoff (in LD) is:

Payoff $=(\mathbf{2 0}-$ your contribution $)+0.6 \cdot$ (total sum of contributions by all players $)$

That means, if, for example, the other two players contribute a total of $70 \mathrm{LD}$ and you contribute $10 \mathrm{LD}$ to the project, then your payoff is

Payoff $=(20-10)+0,6 \cdot(70+10)=58$

Whereas, if the other two players contribute a total of $70 \mathrm{LD}$ and you contribute nothing, your payoff is

Payoff $=(20-0)+0,6 \cdot(70+0)=62$

If you are type30, your payoff is (in LD):

Payoff $=(\mathbf{3 0}-$ your contribution $)+0.6 \cdot($ total sum of contributions by all players $)$

If you are type40, your payoff is (in LD):

Payoff $=(\mathbf{4 0}-$ your contribution $)+0.6 \cdot($ total sum of contributions by all players $)$

In order to ease you the calculation, you will find an Excel file names "simulator" on your screen. For example, you can enter suggestions for your contribution and the contributions of the other group members into the simulator. The payoffs to each player as well as the payoff to the group are calculated.

The game consists of ten separate rounds, in which you always play the same game, you remain the same type and you interact with the same two participants. In each round, you will be informed about the contributions $\left(\mathrm{q}_{1}\right.$ to $\left.\mathrm{q}_{3}\right)$ and the payoffs (payoff ${ }_{1}$ to payoff $\mathrm{f}_{3}$ ) of all players in your group as well as the average values (D).

At the end of the experiment you will receive the payoff of one of the ten rounds in $€(3 \mathrm{LD}=1 €)$. The round that will be disbursed is chosen randomly. Therefore, in each round, you should act as if it was relevant to disbursement. In the beginning, there will be two trial rounds which are not relevant to disbursement.

[Alternative paragraph for rule "Equal Contribution":

The game has two stages. In stage 1 , you decide which minimum contribution, $Q_{\min }$, the group shall make. The other players in your group state as well which minimum contribution, $\mathbf{Q}_{\min }$, they would like to have for the group.

The minimum of the suggestions, $\min \left(\mathbf{Q}_{\mathbf{m i n}}\right)$, is set as the minimum contribution of the group. Then in stage 2, you decide about your contribution, q, to the project, whereby for every player a lower limit, $q_{\text {min }}$, for the individual contribution is calculated from $\min \left(\mathrm{Q}_{\min }\right)$ according to a specific rule.

The following rule will be applied: 
Rule "Equal Contribution": $q_{\min }$ is determined from $\min \left(Q_{\min }\right)$ so that the minimum contributions, $q_{\min }$, of all players are as equal as possible* so that every player contributes at least one third of the group's minimum contribution, $\min \left(\mathrm{Q}_{\min }\right)$, i.e., $\mathrm{q}_{\min }=(1 / 3) \cdot \min \left(\mathrm{Q}_{\min }\right)$.

Examples for the rule "Equal Contribution” with a minimum contribution of the group $\min \left(\mathrm{Q}_{\min }\right)=45^{*}$.

\begin{tabular}{|c|c|c|}
\hline & \multicolumn{2}{|c|}{$\begin{array}{c}\text { Rule } \\
\text { "Equal Contribution" }\end{array}$} \\
\hline & $\begin{array}{c}\text { Lower limit of } \\
\text { contribution }\end{array}$ & Payoff \\
\hline Type20 & 15 & 32 \\
\hline Type30 & 15 & 42 \\
\hline Type40 & 15 & 52 \\
\hline
\end{tabular}

* Assumption: Each player chooses their lower limit as the contribution, i.e. $\mathrm{q}=\mathrm{q}_{\min }$.

[Alternative paragraph for rule "Equal Payoff":

The game has two stages. In stage 1, you decide which minimum contribution, $\mathrm{Q}_{\min }$, the group shall make. The other players in your group state as well which minimum contribution, $\mathbf{Q}_{\min }$, they would like to have for the group.

The minimum of the suggestions, $\min \left(\mathbf{Q}_{\mathbf{m i n}}\right)$, is set as the minimum contribution of the group. Then in stage 2, you decide about your contribution, q, to the project, whereby for every player a lower limit, $q_{\min }$, for the individual contribution is calculated from $\min \left(\mathrm{Q}_{\min }\right)$ according to a specific rule.

The following rule will be applied:

Rule "Equal Payoff": $q_{\min }$ is determined from $\min \left(Q_{\min }\right)$ so that the payoffs off all players are equal or at least adjusted as far as possible**. ]

Examples for the rule "Equal Payoff” with a minimum contribution of the group $\min \left(\mathrm{Q}_{\min }\right)=45^{*}$.

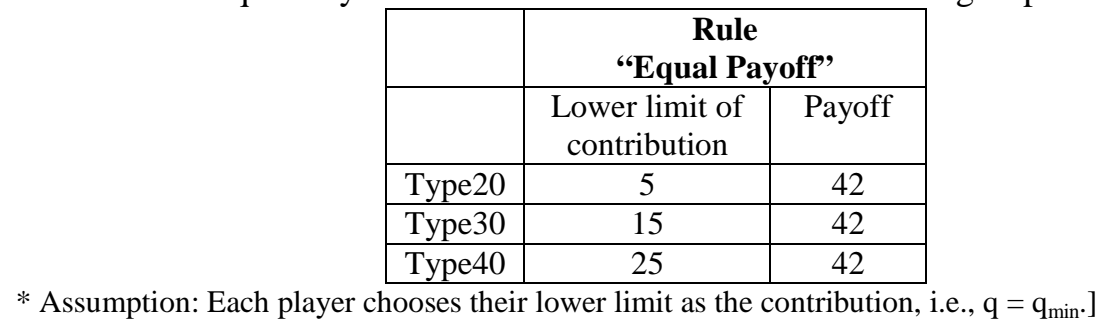

[Alternative paragraph for rule "Proportional Contributions":

The game has two stages. In stage 1, you decide which minimum contribution, $\mathrm{Q}_{\min }$, the group shall make. The other players in your group state as well which minimum contribution, $\mathbf{Q}_{\min }$, they would like to have for the group.

The minimum of the suggestions, $\min \left(\mathbf{Q}_{\min }\right)$, is set as the minimum contribution of the group. Then in stage 2, you decide about your contribution, q, to the project, whereby for every player a lower limit, $q_{\min }$, for the individual contribution is calculated from $\min \left(\mathrm{Q}_{\min }\right)$ according to a specific rule.

The following rule will be applied:

\footnotetext{
* Please note that the adjustment is subject to the condition that the minimum contribution of the group to the joint project is reached.
} 
Rule "Proportional Contribution": $\mathrm{q}_{\min }$ is determined from $\min \left(\mathrm{Q}_{\min }\right)$ so that the minimum contributions, $q_{\min }$, of all players are proportional to their initial endowment. I.e., the higher the initial endowment the higher is the minimum contribution, $\mathrm{q}_{\min }$, to the joint project by the player.]

Examples for the rule "Proportional Contribution" with a minimum contribution of the group $\min \left(\mathrm{Q}_{\min }\right)=$ $45^{*}$.

\begin{tabular}{|c|c|c|}
\hline & \multicolumn{2}{|c|}{$\begin{array}{c}\text { Rule } \\
\text { "Proportional } \\
\text { Contribution” }\end{array}$} \\
\hline & $\begin{array}{c}\text { Lower limit of } \\
\text { contribution }\end{array}$ & Payoff \\
\hline Type20 & 10 & 37 \\
\hline Type30 & 15 & 42 \\
\hline Type40 & 20 & 47 \\
\hline
\end{tabular}

* Assumption: Each player chooses their lower limit as the contribution, i.e., $\mathrm{q}=\mathrm{q}_{\text {min. }}$.]

Please use the simulator to understand the examples. On your screen you will find an Excel file named "simulator". You can enter your desired minimum contribution of the group, $\min \left(Q_{\min }\right)$, in the simulator. For the rule "Equal Contribution" [alternative wording for equal payoff (eqpay) rule: "Equal Payoff"] [alternative wording for the proportional contribution (propcont) rule: "Proportional Contribution"] the individual minimum contributions, $q_{\min }$, and the corresponding payoffs to each player as well as the payoff to the group are calculated. Please note that only the corresponding minimum contributions are calculated, i.e. the minimum contribution of the group, $\min \left(\mathrm{Q}_{\min }\right)$, is distributed to the players according to the different rules. Of course, you can also contribute more than the calculated minimum contribution, but only as long as your contribution does not exceed your initial endowment.

The game consists of ten separate rounds, in which you always play the same game, you remain the same type and you interact with the same two participants. In each round, you will be informed about the proposals of the minimum contribution $\left(\mathrm{Q}_{\min 1}\right.$ to $\left.\mathrm{Q}_{\min 3}\right)$, the contributions $\left(\mathrm{q}_{1}\right.$ to $\left.\mathrm{q}_{3}\right)$ and the payoffs (payoff to payoff $f_{3}$ ) of all players in your group as well as the average values (D).

At the end of the experiment you will receive the payoff of one of the ten rounds in $€(3 \mathrm{LD}=1 €)$. The round that will be disbursed is chosen randomly. Therefore, in each round, you should act as if it was relevant to disbursement. In the beginning, there will be two trial rounds which are not relevant to disbursement.

\section{Control Questions (please answer, use the simulator if necessary)}

[Additional questions for rule: “Equal Contribution”:

1. Assume, the three players have stated 10,20 and 30 respectively as the proposal for the minimum contribution.

What is the group's minimum contribution $\min \left(\mathrm{Q}_{\min }\right)$ ?

The group's minimum contribution, $\min \left(\mathrm{Q}_{\min }\right)$, is:

2. Assume, the group's minimum contribution, $\min \left(\mathrm{Q}_{\min }\right)$, to the project is 30 . What is your minimum contribution and payoff in the "Equal Contribution" rule, if all the players contribute their minimum contribution and you are type20? (Tip: Use the simulator)

\begin{tabular}{|l|c|}
\hline & $\begin{array}{c}\text { Rule } \\
\text { "Equal Contribution” }\end{array}$ \\
\hline My minimum contribution & \\
\hline My payoff & \\
\hline
\end{tabular}

]

[Additional questions for rule "Equal Payoff":

1. Assume, the three players have stated 10, 20 and 30 respectively as the proposal for the minimum contribution.

What is the group's minimum contribution $\min \left(\mathrm{Q}_{\min }\right)$ ?

The group's minimum contribution, $\min \left(\mathrm{Q}_{\min }\right)$, is: 
2. Assume, the group's minimum contribution, $\min \left(\mathrm{Q}_{\min }\right)$, to the project is 30 . What is your minimum contribution and payoff in the "Equal Payoffs" rule, if all the players contribute their minimum contribution and you are type20? (Tip: Use the simulator)

\begin{tabular}{|l|c|}
\hline & $\begin{array}{c}\text { Rule } \\
\text { "Equal Payoffs" }\end{array}$ \\
\hline $\begin{array}{l}\text { My minimum contribution } \\
\mathrm{q}_{\min }\end{array}$ \\
\hline My payoff & \\
\hline
\end{tabular}

]

[Additional question for rule "Proportional Contribution":

1. Assume, the three players have stated 10,20 and 30 respectively as the proposal for the minimum contribution.

What is the group's minimum contribution $\min \left(\mathrm{Q}_{\min }\right)$ ?

The group's minimum contribution, $\min \left(\mathrm{Q}_{\min }\right)$, is:

2. Assume, the group's minimum contribution, $\min \left(\mathrm{Q}_{\min }\right)$, to the project is 30 . What is your minimum contribution and payoff in the "Proportional Contributions" rule, if all the players contribute their minimum contribution and you are type20? (Tip: Use the simulator)

\begin{tabular}{|l|l|}
\hline & $\begin{array}{c}\text { Rule } \\
\text { "Proportional } \\
\text { Contributions" }\end{array}$ \\
\hline $\begin{array}{l}\text { My minimum contribution } \\
\mathrm{q}_{\text {min }}\end{array}$ & \\
\hline My payoff & \\
\hline
\end{tabular}

]

1. Assume that your contribution as Type 40 to the joint project is $20 \mathrm{LD}$. The contributions of the two other group members are 0 and $10 \mathrm{LD}$. What is your payoff?

My payoff is

2. Assume that your contribution as a Type20 to the joint project is $0 \mathrm{LD}$. The contributions of the two other group members are 10 and $20 \mathrm{LD}$. What is your payoff?

My payoff is

3. Assume all the other players in your group have contributed their whole initial endowment to the project. Which of the following contributions gives you the highest payoff as a type40?

(please tick)
O 0 LD
O $10 \mathrm{LD}$
O 20 LD
O $40 \mathrm{LD}$

4. Assume all the other players in your group have contributed their whole initial endowment to the project. Which of the following contributions gives the group the highest payoff if you are a type40? (please tick)
O 0 LD
O $10 \mathrm{LD}$
O $20 \mathrm{LD}$
O $40 \mathrm{LD}$

If you have answered all the questions, please give us a sign. We will then check your answers. The game will start (with explanations on the screen) when all participants have answered the control questions correctly.

Good luck!

The MaXLab-Team 


\section{Appendix F: Screenshots}

Figure F.1: Screenshot of the Collective-Choice Phase of the Multi-Phase-Game (majority voting)

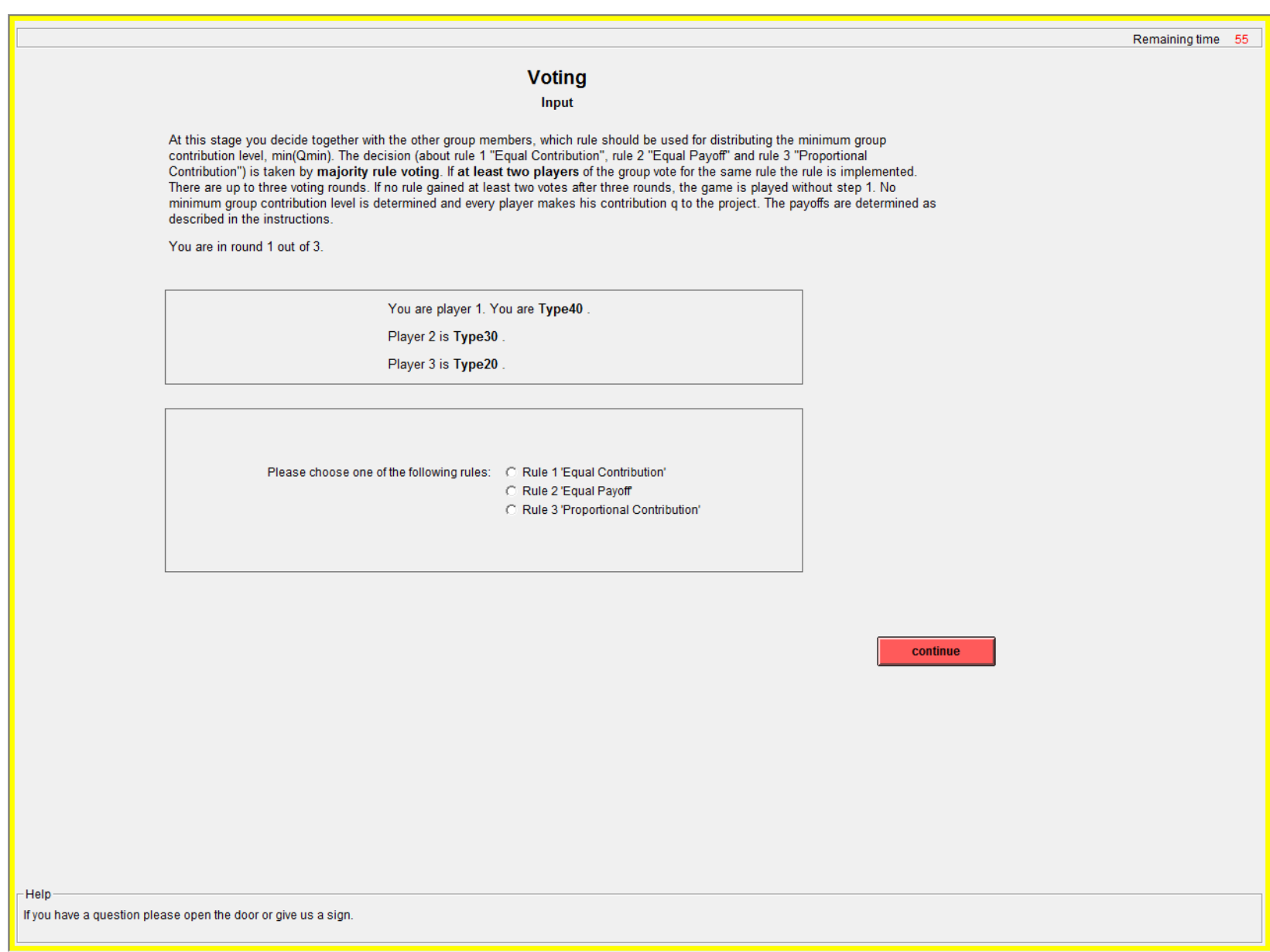


Figure F.2: Screenshot of the Contribution Phase of the Multi-Phase-Game (eqcont)

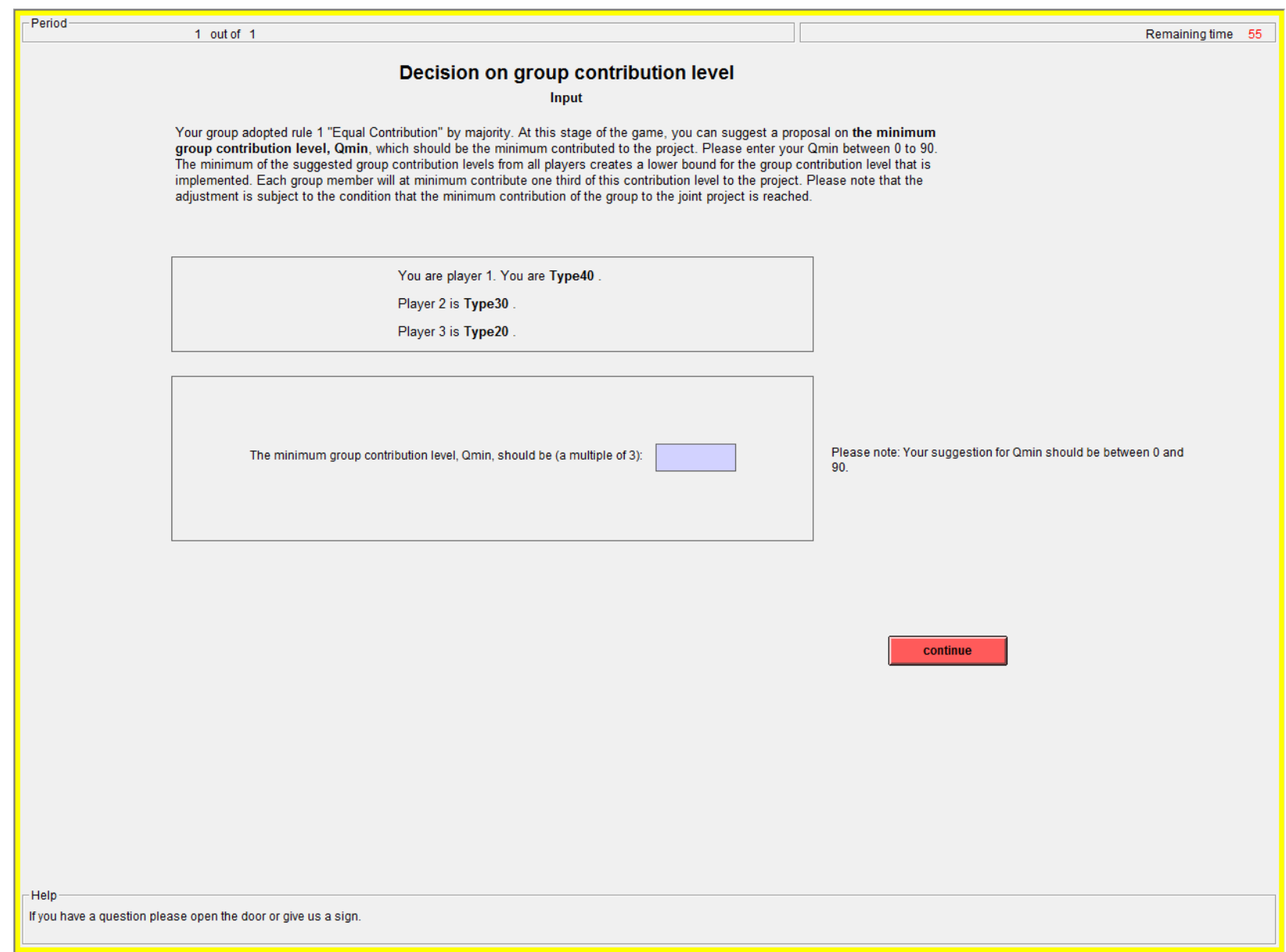


Figure F.3: Screenshot of the Simulator (for $Q_{\min }=45$, above: majority voting, below: propcont)

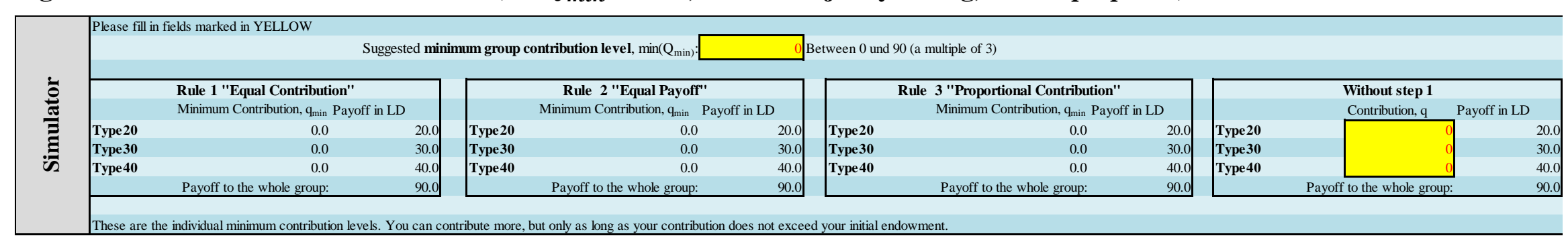

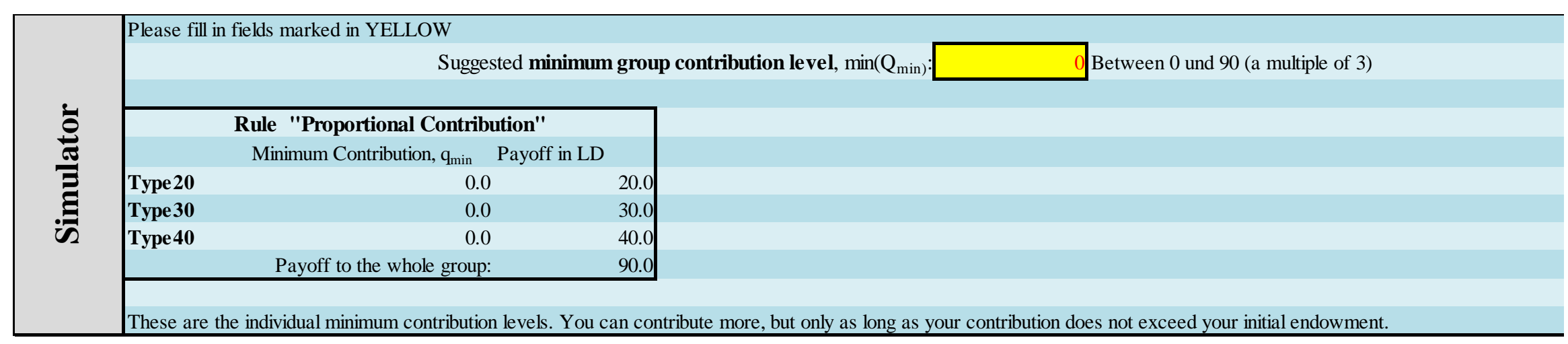

